Article

\title{
EEG Waveform Analysis of P300 ERP with Applications to Brain Computer Interfaces
}

\author{
Rodrigo Ramele *(1), Ana Julia Villar and Juan Miguel Santos ${ }^{\dagger}$ \\ Computer Engineering Department, Instituto Tecnológico de Buenos Aires (ITBA), Buenos Aires 1441, \\ Argentina; jvillar@itba.edu.ar (A.J.V.); jsantos@itba.edu.ar (J.M.S.) \\ * Correspondence: rramele@itba.edu.ar \\ † Current address: C1437FBH Lavarden 315, Ciudad Autónoma de Buenos Aires 1441, Argentina.
}

Received: 27 September 2018; Accepted: 13 November 2018; Published: 16 November 2018

\begin{abstract}
The Electroencephalography (EEG) is not just a mere clinical tool anymore. It has become the de-facto mobile, portable, non-invasive brain imaging sensor to harness brain information in real time. It is now being used to translate or decode brain signals, to diagnose diseases or to implement Brain Computer Interface (BCI) devices. The automatic decoding is mainly implemented by using quantitative algorithms to detect the cloaked information buried in the signal. However, clinical EEG is based intensively on waveforms and the structure of signal plots. Hence, the purpose of this work is to establish a bridge to fill this gap by reviewing and describing the procedures that have been used to detect patterns in the electroencephalographic waveforms, benchmarking them on a controlled pseudo-real dataset of a P300-Based BCI Speller and verifying their performance on a public dataset of a BCI Competition.
\end{abstract}

Keywords: electroencephalography; brain-computer interfaces; waveform; p300; SIFT; PE; MP; SHCC

\section{Introduction}

Current society is demanding technology to provide the means to realize the utopia of social inclusion for people with disabilities [1]. Additionally, as societies are aging [2] the incidence of neuromuscular atrophies, strokes and other invalidating diseases is increasing. Concurrently, the digital revolution and the pervasiveness of digital gadgets have modified the way people interact with the environment through these devices [3]. All this human computer interaction is based on muscular movement [4], but these trends are pushing this boundary beyond the confines of the body and beyond the limitation of human motion. A new form of human machine communication which directly connects the Central Nervous System (CNS) to a machine or computer device is currently being developed: Brain Machine Interfaces (BMI), Brain Computer Interfaces (BCI) or Brain-Neural Computer Interfaces (BNCI).

At the center of all this hype, we can find a hundredth year old technology, rock-solid as a diagnosis tool, which greatly benefited from the shrinkage of sensors, the increase in computer power and the widespread development of wireless protocols and advanced electronics: the Electroencephalogram (EEG) [5].

EEG sensors are wearable [6] non-invasive, portable and mobile [7], with excellent temporal resolution, and acceptable spatial resolution [8]. This humble diagnosis device is been transformed into currently the best approach to detect, out-of-the lab in an ambulatory context, information from the Central Nervous System and to use that information to volitionally drive cars, steer drones, write emails, control wheelchairs or to assess alcohol consumption [9-12].

The clinical and historical tactic to analyze EEG signals is based on detecting visual patterns out of the EEG trace or polygraph [8]: multichannel signals are extracted and continuously plotted 
over a piece of paper. Electroencephalographers or Electroencephalography technician decode and detect patterns along the signals by visually inspecting them [5]. Nowadays clinical EEG still remains a visually interpreted test [8].

The need of quantitative procedures to automate the decoding of EEG signals has been materialized in BCI where around $71.2 \%$ is based on noninvasive EEG [4]. However, methods of decoding signals based on the detection of waveforms has been scarce. Hence, the traditional and knowledgeable approach has been neglected particularly in BCI Research. We aim to help fix this gap by providing a review of the methods which emphasize the waveform, the shape of the EEG signal and which can decode them in a supervised and semi-automated procedure.

The aim of this study is threefold: first to review current literature of EEG processing techniques which are based on analysis of the waveform. The second is to evaluate and study these methods by analyzing its classification performance against a pseudo-real dataset. And third, to verify their applicability to a real and public dataset.

This article unfolds as follows: Section 2 provides a brief introduction to EEG and the particularities of the EEG waveform characterization. Section 3.1 explains the waveform-based algorithms that are analyzed. In Section 3.6 the experimentation procedure is explained. Results are presented in Section 4 and finally Discussion and Conclusions are expounded in the final sections.

\section{Electroencephalography}

The Electroencephalography consists on the measurement of small variations of electrical voltage over the scalp. It is one of the most widespread used methods to capture brain signals and was initially developed by Hans Berger in 1924 and has been extensively used for decades to diagnose neural diseases and other medical conditions.

The first characterization that Dr. Berger detected was the Visual Cortical Alpha Wave, the Berger Rythm [13]. He understood that the amplitude and shape of this rhythm was coherently associated to a cognitive action (eyes closing). We should ask ourselves if the research advancement that came after that discovery would have happened if it weren't so evident that the shape alteration was due to a very simple and verifiable cognitive process.

The EEG signal is a highly complex multi-channel time-series. It can be modeled as a linear stochastic process with great similarities to noise [14]. It is measured in microvolts, and those slightly variations are contaminated with heavy endogenous artifacts and exogenous spurious signals. Figure 1 shows $5 \mathrm{~s}$ of a sample 8-channel EEG signal.

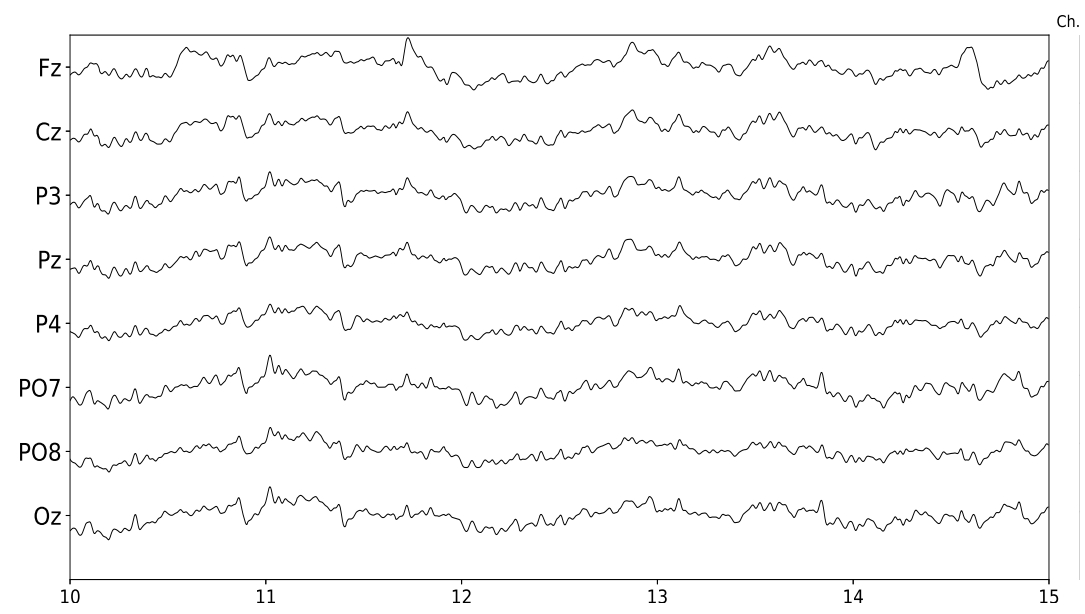

Figure 1. Sample EEG signal obtained from g.Tec g.Nautilus. Time axis is in seconds and five seconds are displayed. The eight channels provided by this device are shown.

The device that captures these small variations in potential differences over the scalp is called the Electroencephalograph. Electrodes are located in predetermined positions over the head, usually 
embedded in saline solutions to facilitate the electrophysiological interface and are connected to a differential amplifier with a high gain which allows the measurement of tiny signals. Although initially analog devices were developed and used, nowadays digital versions connected directly to a computer are pervasive. A detailed explanation on the particularities and modeling of EEG can be obtained from [15], and a description of its electrophysiological aspects from [16].

Overall, EEG signals can be described by their phase, amplitude, frequency and waveform. The following elements regularly characterize EEG signals:

- Artifacts: These are signal sources which are not generated from the CNS, but can be detected from the EEG signal. They are called endogeneous or physiological when they are generated from a biological source like face muscles, ocular movements, etc., and exogeneous or non-physiological when they have an external electromagnetic source like line induced currents or electromagnetic noise [17].

- Non-Stationarity: the statistical parameters that describe the EEG as a random process are not conserved through time, i.e., its mean and variance, and any other higher-order moments are not time-invariant [13].

- DC drift and trending: in EEG jargon, which is derived from concepts of electrical amplifiers theory, Direct Current (DC) refers to very low frequency components of the EEG signal which varies around a common center, usually the zero value. DC drift means that this center value drifts in time. Although sometimes considered as a nuisance that needs to get rid of, it is known that very important cognitive phenomena like Slow Cortical Potentials or Slow Activity Transients in infants do affect the drift and can be used to understand some particular brain functioning [5].

- Basal EEG activity: the EEG is the compound summation of myriads of electrical sources from the CNS. These sources generate a baseline EEG which shows continuous activity with a small or null relation with any concurrent cognitive activity or task.

- Inter-subject and intra-subject variability: EEG can be affected by the person's behavior like sleep hygiene, caffeine intake, smoking habit or alcohol intake previously to the signal measuring procedure [18].

Regarding how the EEG activity can be related to an external stimulus that is affecting the subject, it can be considered as

- $\quad$ Spontaneous: generally treated as noise or basal EEG.

- Evoked: activity that can be detected synchronously after some specific amount of time after the onset of the stimulus. This is usually referred as time-locked. In contrast to the previous one, it is often called Induced activity.

Additionally, according to the existence of a repeated rhythm, the EEG activity can be understood as

- Rhythmic: EEG activity consisting in waves of approximately constant frequency. It is often abbreviated RA (regular rythmic activity). They are loosely classified by their frequencies, and their naming convention was derived from the original naming used by Hans Berger himself, and after Alpha Waves $(10 \mathrm{~Hz})$, it came Delta $(4 \mathrm{~Hz})$, Theta $(4-7 \mathrm{~Hz})$, Sigma $(12-16 \mathrm{~Hz})$, Beta $(12-30 \mathrm{~Hz})$ and Gamma $(30-100 \mathrm{~Hz})$.

- Arrhythmic: EEG activity in which no stable rhythms are present.

- Dysrhythmic: Rhythms and/or patterns of EEG activity that characteristically appear in patient groups and rarely seen in healthy subjects.

The number of electrodes and their positions over the scalp determines a Spatial Structure: signal elements can be generalized, focal or lateralized, depending on in which channel (i.e., electrode) they are found. 


\section{EEG Waveform Characterization}

The shape of the signal, the waveform, can be defined as the graphed line that represents the signal's amplitude plotted against time. It can also be called EEG biomarker, EEG pattern, signal shape, signal form and a morphological signal [13].

The signal context is crucial for waveform characterization, both in a spatial and in a temporal domain [13]. Depending on the context, some specific waveform can be considered as noise while in other cases is precisely the element which has a cognitive functional implication.

A waveform can have a characteristic shape, a rising or falling phase, a pronounced plateau or it may be composed of ripples and wiggles. In order to describe them, they are characterized by its amplitude, the arch, whether they have (non)sinusoidal shape, by the presence of an oscillation or imitating a sawtooth (e.g., Motor Cortical Beta Oscillations). The characterization by their sharpness is also common, particularly in Epilepsy, and they can also be identified by their resemblance to spikes (e.g., Spike-wave discharge).

Depictions may include subjective definitions of sharper, arch comb or wicket shape, rectangular, containing a decay phase or voltage rise, peaks and troughs, short term voltage change around each extrema in the raw trace. Derived ratios and indexes can be used as well, like peak and trough sharpness ratio, symmetry between rise and decay phase and slope ratio (steepness of the rise period to that of the adjacent decay period). For instance, wording like "Central trough is sharper and more negative that the adjacent troughs" [19] are common in the literature.

Other regular characterizations which are based on the waveform shape may encompass:

- Attenuation: Also called suppression or depression. Reduction of amplitude of EEG activity resulting from decreased voltage. When activity is attenuated by stimulation, it is said to have been "blocked" or to show "blocking".

- Hypersynchrony: Seen as an increase in voltage and regularity of rhythmic activity, or within the alpha, beta, or theta range. The term suggest an increase in the number of neural elements contributing to the rhythm, or in the synchronization of different neurons with the same discharge pattern [20].

- $\quad$ Paroxysmal: Activity that emerges from background with a rapid onset, reaching frequently high voltage and ending with an abrupt return to lower voltage activity.

- Monomorphic: Activity appearing to be composed of one dominant waveform pattern.

- Polymorphic: Activity composed of multiple frequencies that combine to form a complex waveform.

- Transient/Component: An isolated wave or pattern that is distinctly different from background activity.

The traditional clinical approach to study electroencephalographic signals consists in analyzing the paper strip that is generated by the plot of the signal obtained from the device. Expert technician and physicians analyze visually the plots looking for specific patterns that may give a hint of the underlying cognitive process or pathology. Atlases and guidelines were created in order to help in the recognition of these complex patterns. Video-electroencephalography scalp recordings are routinely used as a diagnostic tools [21]. The clinical EEG research has also focused on temporal waveforms, and a whole branch of electrophenomenology has arisen around EEG graphoelements [5].

Sleep Research has been studied in this way by performing Polysomnographic recordings (PSG) [22,23]. The different sleep stages are evaluated by visually marking waveforms or graphoelements in long-running electroencephalographic recordings, looking for patterns based on standardized guidelines [24]. Visual characterization includes the identification or classification of certain waveform components based on a subjective characterization (e.g., positive or negative peak polarity) or the location within the strip. It is regular to establish an amplitude difference between different waveforms from which a relation between them is reckoned and a structured index is created (e.g., sleep K-Complex is well characterized based on rates between positive vs negative 
amplitude) [25]. Other relevant EEG patterns for sleep stage scoring are alpha, theta, and delta waves, sleep spindles, polysplindles, vertex sharp waves (VSW), and sawtooth waves (REM Sleep).

Moreover, EEG data acquisition is a key procedure during the assessment of patients with focal Epilepsy for potential seizure surgery, where the source of the seizure activity must be reliably identified. The onset of the Epileptic Seizure is defined as the first electrical change seen in the EEG rhythm which can be visually identified from the context and it is verified against any clinical sign indicating seizure onset. The Interictal Epileptiform Discharges (IEDs) are visually identified from the paper strip, and they are also named according to their shape: spike, spike and wave or sharp-wave discharges [26].

Waveform characterization is the method in which analysis has been performed for Event Related Potentials (ERP). These are transient signal elements that may arise as a brain response to an external visual, tactile or auditory stimulus. ERPs are regularly used to assess auditory response in infants. They are extensively used and studied in Cognitive Neuroscience [27]. ERPs are identified by their components which are recognizable signal shapes assigned to the observed waveform, that can be linked to some cognitive or measurable psychological process. One of the most studied ERP is the P300, discovered in 1965 by Sutton, Braren, Zubin and John. This component is a positive deflection of a subject's EEG signal that arises when an unexpected and infrequent stimulus appears [1]. The P300 is widely utilized in BCI because it can be harnessed to implement a Speller application. Hence, P300 ERPs are a target phenomena to study by automatic waveform recognition methods.

Table 1 summarizes a list of depictions used to describe waveforms in the surveyed literature. Epilepsy has been described by the nature of oscillatory characterization of their waves, like ripples and wiggles, imitating sawtooths or by their geometric shape. For ERPs on the other hand, more elaborate indexes has been provided, establishing relations between amplitudes of signal components. Finally, Sleep studies and ICU research are areas where the most complex indexes has been derived, particularly the coupling of signal properties like phase, amplitude and frequency.

Table 1. EEG waveforms descriptions found in the surveyed literature.

\begin{tabular}{ccc}
\hline Method & Phenomena & Reference \\
\hline Positive Rounded Component & $\alpha$-Waves, Epilepsy & {$[5,28]$} \\
Rising and Falling Phase & Epilepsy & {$[14,28]$} \\
Terminal plateau & Epilepsy & {$[14]$} \\
Ripples and Wiggles & Epilepsy, ERP & {$[14,26,29,30]$} \\
Sinusoidal Shape & Epilepsy & {$[19,28-31]$} \\
Sawtooth & Motor Imagery, Sleep & {$[22,26,28]$} \\
Sharpness or Spike-like & Epilepsy & {$[8,14,26,32]$} \\
Rectangular & Epilepsy & {$[14,19]$} \\
Line length & Anomaly Detection & {$[33]$} \\
Root Mean Square & Anomaly Detection & {$[33]$} \\
Wicket Shape & Epilepsy & {$[5,8,19,26,28,32]$} \\
Peak and Trough Sharpness Ratio & Epilepsy & {$[8,19,32,34]$} \\
Symmetry between rise and decay phase & Epilepsy & {$[8,19]$} \\
Slope Ratio & Sleep & {$[35]$} \\
Positive/Negative Peak Amplitude & ERP & {$[8,14,19,28,36,37]$} \\
Positive vs Negative Ratio & Sleep K-Complex & {$[26]$} \\
Base-to-Peak Amplitude & ERP & {$[19]$} \\
Peak-to-Peak Amplitude & ERP & {$[33,36]$} \\
Positive/Negative Peak Latency & ERP & {$[36]$} \\
Integrated Activity & ERP, Epilepsy, ICU & {$[25,33,38]$} \\
Cross-Correlation & ERP, Epilepsy, Sleep & {$[29,38]$} \\
Coupling & & \\
Period Amplitude Analysis & Sleep & {$[19]$} \\
Cross Frequency, Phase-Amplitude, Phase-Phase & ERP, Epilepsy & {$[25,29,38]$} \\
\hline
\end{tabular}




\section{Materials and Methods}

The exploration of methods based on waveforms is conducted by following the PRISMA [39] guidelines. Search is performed on Google Scholar, Semantic Web and IEEE Xplore search engines by the terms "Waveforms" OR "Shape" OR “Morphology" OR "Visual inspection" + "EEG".

The following criteria is proposed to identify methods which are based on the signal's waveform:

1. The analysis considers the shape of the plot of the signal.

2. The pattern can be identified and verified by visual inspection.

3. The pattern matching is performed in time-domain.

4. The method encompass a feature extraction procedure.

5. The feature extraction procedure allows to create a template dictionary.

As described in [40] the Pattern Matching problem in Signal processing is finding a signal given the region that best describes the structure of the prototype signal template. On the other hand, a feature is a meaningful quantification, usually a multidimensional vector, that synthesize the information of a given signal or signal segment [41].

\subsection{EEG Waveform Analysis Algorithms}

Shape or waveform analysis methods are considered as nonparametric methods. They explore signal's time-domain metrics or even derive more complex indexes or features from it [42].

One of the earliest approach to automatically process EEG data is the Peak Picking method. Although of limited usability, peak picking has been used to determine latency of transient events in EEG $[43,44]$. Straightforward in its implementation, it consists in assigning a component to a simple waveform element based on the expected location of its more prominent deflection [31]. Of regular use in ERP Research, the name of many of the EEG features reference directly a peak within the component, e.g., P300 or P3a P3b or N100. This leads to a natural way to classify them visually by selecting appropriate peaks and matching their positions and amplitudes in an orderly manner. The letter provides the polarity (Positive or Negative) and the numbering shows the time referencing the stimulus onset, or the ordinal position of each peak (first, second, etc).

A related method is used in [45] where the area under the curve of the EEG is sumarized to derive a feature. This was even used in the seminal work of Farwell and Donchin on the P300 Speller [41,46]. Additionally, a logarithmic graph of the peak-to-peak amplitude which is called amplitude integrated EEG (aEEG) [38] is used nowadays in Neonatal Intensive Care Units.

Other works on EEG explored the idea to extend human capacities analyzing EEG waveforms. In [47] a feature derived from the amplitude and frequency of its signal and its derivative in time-domain is used. Moreover, Yamaguchi et al. [48] explored the use of Mathematical Morphology, where the time-domain structure of contractions and dilations were studied. Finally the proposals of Burch, Fujimori, Uchida and the Period Amplitude Analysis (PAA) [49] algorithm are few of the earliest depictions where the idea of capturing the shape of the signal were established.

According to the defined criteria, the algorithms that will be evaluated are as follows:

- Matching Pursuit

- Permutation Entropy

- $\quad$ Slope Horizontal Chain Code

- $\quad$ Scale Invariant Feature Transform

All these methods provide a feature $f$ that can be used as a template. The notation $f=\left\{f_{i}\right\}_{1}^{n}$ or $f=\left\{f_{i}\right\}_{i \in J}$ is used to describe the concatenation of scalar values to form a multidimensional feature vector $f=\left\{f_{1}, f_{2}, \ldots, f_{n}\right\}$. These algorithms are all based on metrics that are extracted from the shape of the single channel digital EEG signal $x(n)$, with $n$ varying from 1 to the length $N$ of the EEG segment in sample points. These features are used to create dictionaries or template databases. Finally, these templates provide the basis for the pattern matching algorithm and offline classification. 
Algorithms were implemented on MATLAB 2014a (Mathworks Inc., Natick, MA, USA). To maintain reproducibility, the dataset described in Section 3.6.1 and the source code has been made available in the online repository of the Code Ocean platform under the name EEGWave.

\subsection{Matching Pursuit-MP 1 and MP 2}

Pursuit algorithms refer, in their many variants, as blind source separation [50] techniques that assume the EEG signal as a linear combination of different sparse sources extracted from a template's dictionaries. Matching Pursuit MP [51], the most representative of these algorithms, is a greedy variant that decomposes a signal into a linear combination of waveforms, called atoms, that are well localized in time and frequency [52]. Given a signal, this optimization technique, tries to find the indexes of $m$ atoms and their weights (contributions) that minimize,

$$
\varepsilon=\left\|x(n)-\sum_{i=1}^{m} w_{i} g_{i}(n)\right\|
$$

which is the error between the signal and its approximation constructed by the weighted $w_{i}$ atoms $g_{i}$, and calculating the euclidean norm $\|\cdot\|_{2}$. The algorithm goes by first setting the approximating signal $\tilde{x}_{0}$ as the original signal itself,

$$
\tilde{x}_{0}(n)=x(n)
$$

and setting the iterative counter $k$ as 1 . Hence, it searches recurrently the best template out of the dictionary that matches current approximation.

$$
g_{k}=\arg \max _{g_{i}}\left|\sum_{n=1}^{N} \tilde{x}_{k-1}(n) g_{i}(n)\right|
$$

where $g_{i}$ are all the available scaled, translated and modulated atoms from the dictionary. The operation $|\cdot|$ corresponds to the absolute value of the inner product. This step determines the atom selection process, and their contribution is calculated based on

$$
w_{k}=\frac{\sum_{n=1}^{N} \tilde{x}_{k-1}(n) g_{k}(n)}{\left\|g_{k}\right\|^{2}}
$$

with $k$ representing the index of the selected atom $g_{k}$ and $\|\cdot\|_{2}$ its euclidean norm. Finally the contribution of each atom is subtracted from the next approximation $[32,51,53]$

$$
\tilde{x}_{k}(n)=\tilde{x}_{k-1}(n)-w_{k} g_{k}(n)
$$

The stopping criteria can be established based on a limiting threshold on Equation (1) or based on a predetermined number of steps and selected atoms. Two variants of this algorithm are evaluated. In MP 1 the dictionary is constructed with the normalized templates directly extracted from the real signal segments which is a straightforward implementation of the pattern matching technique. In $M P$ 2 the coefficients of Daubechies least-asymetric wavelet with 2 vanishing moments atoms are used to construct the dictionary [54]. For the first version, the matching against the template is evaluated according to Equation (1) directly, whereas for the latter each feature is crafted by decomposing the signal in its coefficients and building, an eventually sparse, vector with them:

$$
f=\left\{w_{i}\right\}_{1}^{D}
$$

where $D$ is the size of the dictionary. 


\subsection{Permutation Entropy—PE}

Bond and Pompe Permutation Entropy has been extensively used in EEG processing, with applications on Anesthesia, Sleep Stage evaluation and increasingly for Epilepsy pre-ictal detection [55]. This method generates a code based on the orderly arrangement of sequential samples, and then derives a metric which is based on the number of times each sequence is found along the signal. This numeric value can be calculated as information entropy [56]. Let's consider a signal on a window of length $W$ represented by the sample points

$$
\left(x_{1}, x_{2}, \ldots, x_{W}\right)
$$

and resampled by $\tau$ intervals, starting from the sampling point $n$, doing

$$
\left(x_{n}, x_{n+\tau}, x_{n+2 \tau} \ldots, x_{n+(m-1) \tau}\right) \text {. }
$$

This sequence is of order $m$, which is the number of sample points used to derive the ordinal element called $\pi$. There are $m$ ! ways in which this sequence can be orderly arranged, according to the position that each sample point holds within the sequence in a decreasing order relationship [57]. For example if $m=3$, and the first sample point is the bigger, the second is the smaller and the third one is in the middle, the ordinal element $\pi$ corresponds to $(1,3,2)$. Thus, along the signal window there can be at most $k$ different ordinal (and overlapping) elements $\pi_{s}$

$$
\left(\pi_{1}, \pi_{2}, \ldots, \pi_{k}\right)
$$

with $k=W-(m-1) \tau$. The probability density function $p d f$ for all the available permutations of order $m$ should be $\mathbf{p}=\left(p_{1}, p_{2}, \ldots, p_{m !}\right)$ with $\sum_{i=1}^{m !} p_{i}=1$.

Hence, the time series window is mapped to a new set of $k$ ordinal elements, and the $p d f$ can be calculated by the empirical permutation entropy,

$$
p_{i}=\frac{1}{k} \sum_{s=1}^{k}\left[\pi_{s}=\pi_{i}\right]
$$

with $1 \leq i \leq m$ !. The Iverson Bracket $[\cdot]$ resolves to 1 when their logical proposition argument is true, 0 otherwise. Therefore, for each $i$ only those ordinal elements $\pi_{s}$ that were effectively found along the signal are counted to estimate $p_{i}$, and zero elsewhere. The empirical permutation entropy can be calculated from the histogram as,

$$
H(\mathbf{p})=\sum_{i=1}^{m !} p_{i} \log \frac{1}{p_{i}} .
$$

The implemented code was derived from [58], and the model description from [59]. This procedure produces a scalar number for the given signal window of size $W$. To derive a feature, a sliding window procedure must be implemented to cover an entire segment of length $N$. Thus, the length of the feature is $N-(W+\tau(m-1))$.

$$
f=\left\{H(\mathbf{p})_{u}\right\}_{W+\tau m}^{N} .
$$

with $u$ varying on a sample by sample basis along the signal, starting from the specified index.

\subsection{Slope Horizontal Chain Code-SHCC}

This algorithm [45] proceeds by generating a coding scheme from a sequence of sample points. This encoding is based on the angle between the horizontal line on a 2D-plane and any segment produced by two consecutive sample points, regarding them as coordinates on that plane. 
A signal of length $N$, can be represented by a list of ordered-pairs $e$,

$$
e=\left[(x, y)_{1},(x, y)_{2}, \ldots,(x, y)_{N}\right]
$$

and it can be divided into $G$ different blocks. These blocks are obtained by resampling the original signal from the index

$$
G=\lfloor n+(m \Delta)+0.5\rfloor
$$

with $n$ being the original sampling index on $1 \leq n \leq N$ and $\lfloor\cdot\rfloor$ being the floor operation, i.e., rounding of the number argument to the closest smaller integer number. On the other hand, $\Delta$ can be obtained by

$$
\Delta=\left\lceil\frac{N}{G+1}\right\rceil
$$

with $G<N$ and using instead $\lceil\cdot\rceil$ as the ceil operation, the rounding to the closest bigger integer number. Lastly, the value $m$ can be derived from

$$
m=\operatorname{sign}\left(\frac{N-1}{\Delta}\right)\left\lfloor\left|\frac{N-1}{\Delta}\right|\right\rfloor .
$$

This resampling produces a new sequence of values,

$$
e^{\prime}=\left[\left(x^{\prime}, y^{\prime}\right)_{1}, \ldots,\left(x^{\prime}, y^{\prime}\right)_{s}, \ldots,\left(x^{\prime}, y^{\prime}\right)_{G}\right]
$$

The next step is the normalization of each ordered-pair as vectors $\mathbf{x}^{\prime}=\left(x_{1}^{\prime}, \ldots, x_{G}^{\prime}\right)$ and $\mathbf{y}^{\prime}=$ $\left(y_{1}^{\prime}, \ldots, y_{G}^{\prime}\right)$ according to

$$
\begin{aligned}
& \hat{\mathbf{x}}=\frac{\mathbf{x}^{\prime}-\min \left(\mathbf{x}^{\prime}\right) \mathbf{1}}{\max \left(\mathbf{x}^{\prime}\right)-\min \left(\mathbf{x}^{\prime}\right)} \\
& \hat{\mathbf{y}}=\frac{\mathbf{y}^{\prime}-\min \left(\mathbf{y}^{\prime}\right) \mathbf{1}}{\max \left(\mathbf{y}^{\prime}\right)-\min \left(\mathbf{y}^{\prime}\right)}
\end{aligned}
$$

with 1 being the vector of length $G$ with all their components equal to 1 . Hence, the scalar components $\hat{x}_{s}$ of $\hat{\mathbf{x}}$, and $\hat{y}_{s}$ of $\hat{\mathbf{y}}$, with $s$ varying between 1 and $G$ are effectively normalized to $\hat{x}_{s}, \hat{y}_{s} \in[0,1]$.

Finally, the feature is constructed by calculating the point-to-point slope against the horizontal plane,

$$
f=\left\{\frac{\hat{y}_{s}-\hat{y}_{s-1}}{\hat{x}_{s}-\hat{x}_{s-1}}\right\}_{2}^{G}
$$

\subsection{Scale Invariant Feature Transform-SIFT}

SIFT [60] is a very successful feature extraction technique from Computer Vision. It has a biomimetic inspiration on how the visual cortex analyze images based on orientations [61]. This method has been used in [62] to analyze EEG signals based on their plots on 2D images.

The first step of the algorithm is the plot generation based on single-channel EEG segments $x(n)$. Hence, this signal is normalized by the z-score [63]:

$$
\tilde{x}(n)=\left\lfloor\frac{\delta(x(n)-\bar{x})}{\sigma_{x}}\right\rceil
$$

with $\delta$ being the signal magnification factor and $\bar{x}$ and $\sigma_{x}$, the mean and standard deviation of $x$ on the signal segment. The width of the image is determined based on the 1-s length size of the segment in 
sample units. This corresponds to the sampling frequency $F_{s}$ of the EEG signal segment. The width is adjusted by multiplying by the magnification factor $\delta$,

$$
w=\delta F_{s}
$$

whereas the height is calculated based on the peak-to-peak amplitude of the signal within the segment,

$$
h=\max _{n} \tilde{x}(n)-\min _{n} \tilde{x}(n) .
$$

Equation (24) determines the vertical position of the image where the signal's zero value will be located.

$$
z=\left\lfloor\frac{h}{2}\right\rfloor-\left\lfloor\frac{\max _{n} \tilde{x}(n)+\min _{n} \tilde{x}(n)}{2}\right\rfloor .
$$

Finally, a binary, black-and-white image plot is generated based on

$$
I\left(z_{1}, z_{2}\right)=\left\{\begin{aligned}
255 & \text { if } z_{1}=\delta n ; z_{2}=\tilde{x}(n)+z \\
0 & \text { otherwise }
\end{aligned}\right.
$$

where $z_{1}$ and $z_{2}$ are the image coordinates values, 255 represents white and 0 is the background black color of the plot. These points are interpolated using the Bresenham algorithm [62].

Once the plot is generated, its center is used to localize the center of the SIFT patch. This region of the image, where the signal's most important salient waveform should be located, is divided in a grid of $4 \times 4$ block and the bidimensional gradient vectors are calculated on each one of them. Therefore, for each block $(i, j)$ within the patch, a histogram $h(i, j, \theta)$ of the gradient orientations, for 8 circular orientations $\theta$, are calculated. This histogram is concatenated for all the 16 blocks and a feature is thus formed:

$$
f=\left\{\left\{\{h(i, j, \theta)\}_{i \in I}\right\}_{j \in I}\right\}_{\theta \in \Theta}
$$

with $i$ and $j$ belonging to $I=\{0,1,2,3\}$ and localizing the 16 blocks within the grid. The angles $\theta$ that belong to $\Theta$ are the eight possible equidistant values between 0 and 315. This vector is normalized, clamped to 0.2 , and re-normalized again. Details of the method can be found on $[60,62]$. It was implemented using the VLFeat [64] public Computer Vision libraries.

\subsection{Experimental Protocol}

The objective of the following experiments is to assess the performance of the algorithms that aim to recognize the shape of the P300 waveform, obtained after averaging signal segments. This performance is evaluated by processing a pseudo-real dataset with two modalities where subtle alterations on the latency and amplitude of the P300 component are simulated in a controlled environment. The experiments are performed by the offline evaluation of the character identification rate of a Visual P300-Based BCI Speller application.

Farwell and Donchin P300 Speller [46,65] is one the most used BCI paradigms to implement a thought translation device and to send commands to a computer in the form of selected letters, similar to typing on a virtual keyboard. This procedure exploits a cognitive phenomena raised by the oddball paradigm [27]: along the EEG trace of a person which is focusing on a sequence of two different visual flashing stimulus, a particular and distinctive transient component is found each time the expected stimulus flashes. This is cleverly utilized in the P300 Speller, where rows and columns of a $6 \times 6$ matrix flashes randomly but only the flashing of a column or row where the letter that a user is focusing will trigger concurrently the P300 ERP along the EEG trace.

A problem with the information produced by a P300 Speller is that the subjects that take part on the experiment are within the closed loop of the BCI system and the human is not a static compliant entity that always performs what the experimenter asks for in a precise and consistent way [66]. 
Therefore, P300 experiments data is often mined with null-signals. These are EEG streams which are marked as having the signal component but, because the subject was not particularly focused, or concentrated, the expected signal element is not generated. This lack of certainty may be in detriment of any conducted analysis and can be misleading or difficult to deal with. Previous works have addressed this same issue, particularly when benchmarking different algorithms $[31,43,67]$.

In order to tackle this problem, a pseudo-real dataset based on an EEG stream is generated under two different modalities. A passive modality and an active modality.

\subsubsection{EEG Stream Generation}

Eight (8) healthy participants are recruited voluntarily and the experiment is conducted anonymously in accordance with the Declaration of Helsinki published by the World Health Organization. No monetary compensation is handed out and they agree and sign a written informed consent. This study is approved by the Departamento de Investigación y Doctorado, Instituto Tecnológico de Buenos Aires (ITBA). The participants are healthy and have normal or corrected-to-normal vision and no history of neurological disorders. These voluntary subjects are aged between 20-40 years old. EEG data is collected in a single recording session. Each subject is seated in a comfortable chair, with her/his vision aligned to a computer screen located one meter in front of her/him. The handling and processing of the data and stimuli is conducted by the OpenVibe platform [68]. Gel-based active electrodes (g.LADYbird, g.Tec, Austria) are used on locations Fz, Cz, Pz, Oz, P3,P4, PO7 and PO8 according to the 10-20 international system. Reference is set to the right ear lobe and ground is preset as the AFz position. Sampling frequency is set to $250 \mathrm{~Hz}$.

The experimental protocol is composed of 35 trials to spell 7 words of 5 letters each. Each trial is composed of 10 intensification sequences of the 6 columns and 6 rows of the Speller Matrix. This yields exactly 120 intensifications of rows and columns per trial. The duration of each intensification as well as the Inter-Stimulus Interval, the pause between stimulations, are set to $0.125 \mathrm{~s}$. This provides a $4 \mathrm{~Hz}$ frequency of flashes on the screen. The initial pause and the inter-trial pauses are set to $20 \mathrm{~s}$. The whole experiment lasts for around $1400 \mathrm{~s}$. This produces an EEG stream which contains 4200 marked sections where 3500 of them are labeled as True and the remaining 700 as False. The extracted EEG signals are band-pass filtered using a 4th order Butterworth digital filter between 0.1 and $10 \mathrm{~Hz}$ and a $50 \mathrm{~Hz}$ notch filter is applied to remove line AC noise. The EEG trace is finally downsampled to $16 \mathrm{~Hz}$. Segments of 1-s length are extracted according to the markers information and those with variations larger than $70 \mu \mathrm{V}$ are identified as artifacts and eliminated.

Four out of the eight participants are instructed to passively watch the flashing screen while not focusing on any particular letter. They do not receive any extra information on the screen. None of them have any experience with a $\mathrm{BCI}$ device. A questionnaire is handed out at the end of the experiment with questions about how the participant felt during it, without giving more details.

The remaining four participants perform a copy-spelling task where the computer monitor highlights the target letter, which is the one that the subject needs to focus. Across the duration of the trial, the current target letter is informed at the bottom of the screen.

\subsubsection{Passive Modality}

First for a passive modality, real P300 ERP templates obtained from a public dataset, are superimposed into the generated EEG stream of 4 subjects. A set of template ERPs is acquired from the Subject Number 8 of the public dataset 008-2014 [69] published on the BNCI-Horizon website [70] by IRCCS Fondazione Santa Lucia. The experimental protocol implemented to produce this dataset is the same as the one described in Section 3.6.1. On the other hand, the EEG traces where these templates are superimposed, are experimentally obtained by subjects which are observing the flashing of the stimulus matrix during a P300 Speller procedure but they do not engage in focusing on any letter in particular. Everything is there, except the P300 ERP component. Hence, along the EEG stream, the markers information is used to localize the True segments where the P300 should be found, 
and those timing locations are used to superimpose the extracted ERP waveform. By implementing this pseudo-real approach, it is possible to effectively control null-signals and to adjust the shape of the evoked potential.

A sample P300 ERP obtained from the trial number 2 of Subject 8 can be seen in Figure 2. These templates are selected due to their shapes more closely resembling the prototypical P300 waveform $[71,72]$. They are produced by extracting segments for this subject and by point-to-point coherently average them.

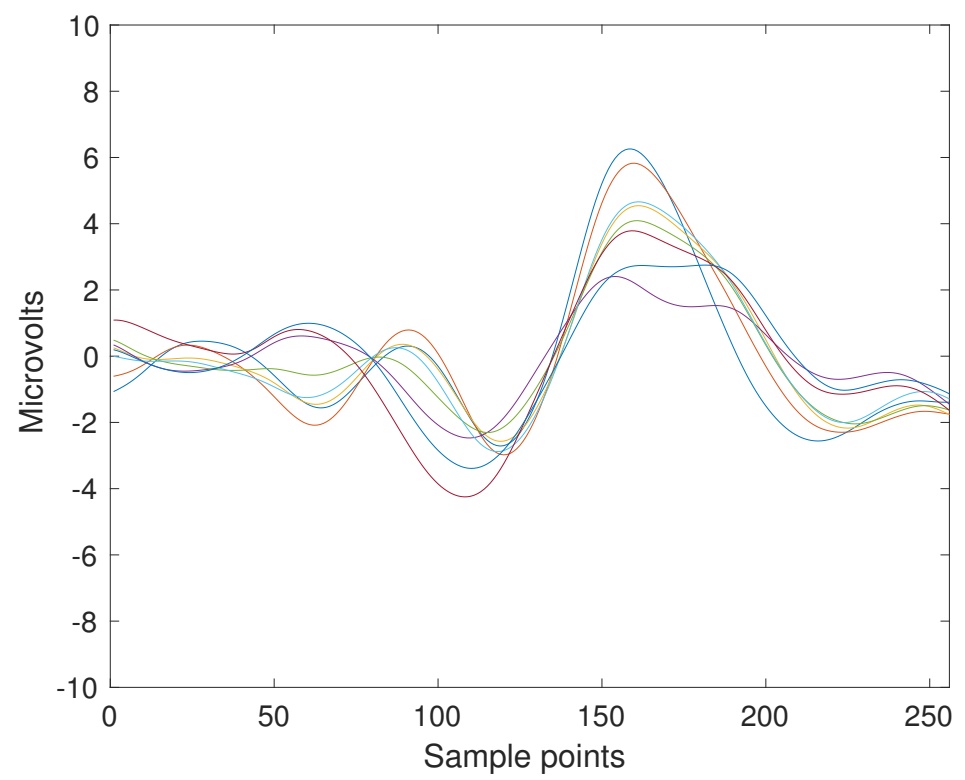

Figure 2. ERP Template obtained from the coherent point-to-point ensemble average from the signals of Subject Number Eight of the BNCI Horizon public dataset 008-2014. The template is $1 \mathrm{~s}$ long which is 256 sample points, and the eight channels are superimposed with different colors. The P3b component can be seen around the sample index 150 and 200.

\subsubsection{Active Modality}

Second, an active modality is also implemented, where a P300-Based BCI Speller experiment is performed on four subjects. For this scenario, the signal segments are modified to guarantee the inclusion of a P300 component. However, in this case the templates are extracted from the same subject. Hence, the EEG signal is preprocessed and labeled segments are extracted. Segments labeled True are coherently point-to-point averaged, and 70 templates are produced from the whole set of 35 trials.

Once templates are procured, a random False segment for the same subject is obtained. This is used as a baseline signal and is added to the template, conforming a new segment which has a superimposed P300 template. This procedure continues until the 700 segments marked as True are completed.

Figure 3 shows a $5 \mathrm{~s}$ sample of the EEG trace obtained with the MNE library [73]. Channel $S$ represents the twelve different stimulus markers (columns or rows) while channel $L$ represent the label (True vs False). Labels are represented by square signals. False segments are marked with single amplitude square signals while True segments are identified by double-amplitude square signals. Subfigure (a) shows the signals before the ERP template is superimposed while subfigure (b) shows the same signals with the superimposed ERP template. At first-sight, differences are really hard to spot visually. Subfigures (c) and (d) show only one second of channels Cz and L from the same segment. The superimposed ERP can be devised enclosed by the vertical bars, around $31.5 \mathrm{~s}$, where in (d) the peak is slightly bigger. Figure 4 shows the obtained ensemble average ERPs as result of superimposing the template signal into the EEG stream, time-locked to the stimulus onset. These 12 point-to-point averaged segments correspond to the first trial of the EEG stream. 


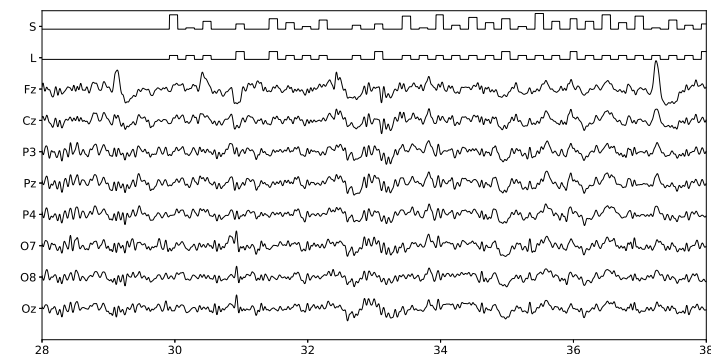

(a) EEG trace of the original signal. The horizontal axis represents $10 \mathrm{~s}$ of the EEG stream, from the 28th second up to the 38 th

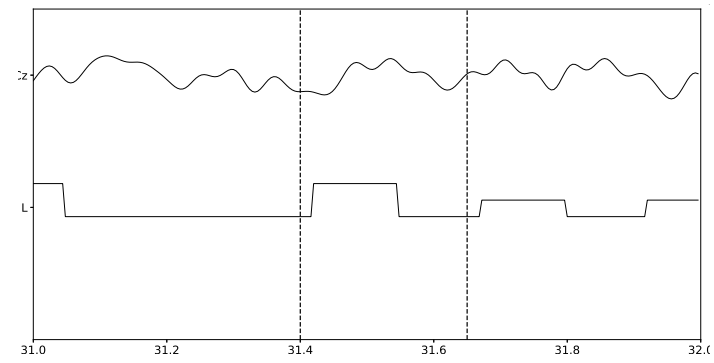

(c) EEG sample of $\mathrm{C} z$ and $\mathrm{L}$ channel of the original EEG trace. Only 1 second is shown here, at the 31 th second.

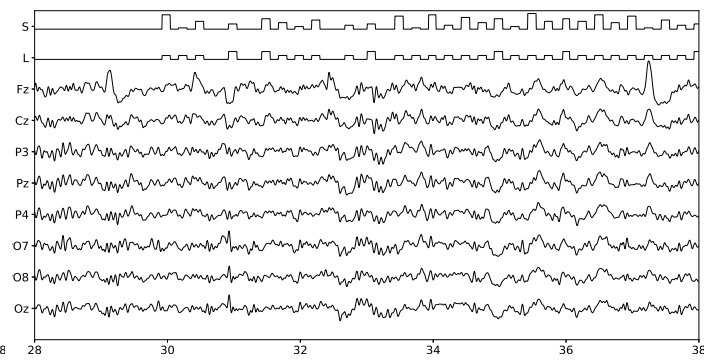

(b) The same 10-s eight-channel signal segment with the superimposed template.

Figure 3. Eight-channel EEG signal for Subject Number 1 of the pseudo-real dataset without and with the superimposed ERP Template. The channel L, the mark which identifies where to superimpose the P300 ERP, is shown as well as the channel S which identifies the stimulus that was presented. On (c,d) the small variation that was introduced by the superimposition of the ERP can be seen enclosed by the vertical bars, where the slope of the bump on subfigure (d) is slightly bigger.

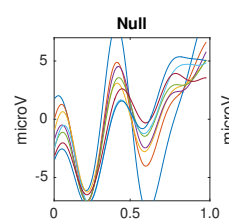

Null

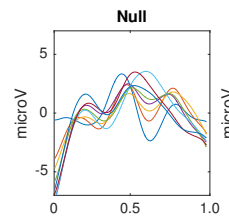

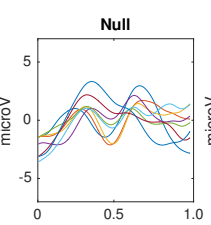

Null

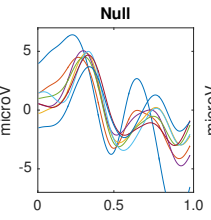

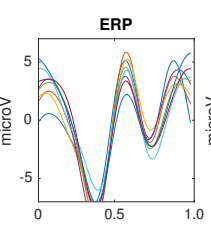

ERP

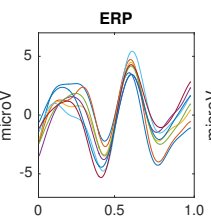

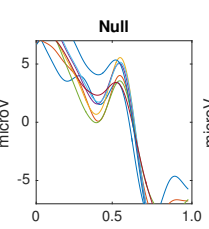

Null

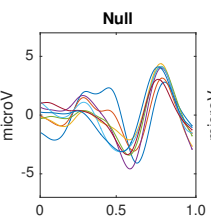

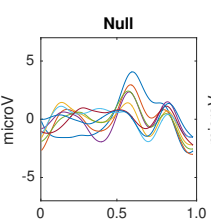

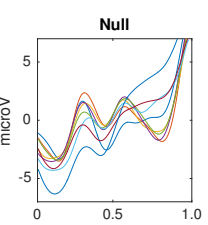

Null
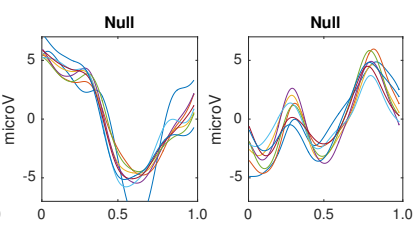

Figure 4. Point-to-point averaged signals. These are extracted from the first letter identification trial of the Subject 1 of the pseudo-real dataset. The ERP is superimposed on classes 3 and 9. Class 3 is obtained while averaging the segments where the row of the speller matrix is intensified whereas class 9 is calculated from the intensification of the corresponding column.

\subsubsection{Experiments}

The experiments are as follows:

- Experiment 1-Letter Identification Performance: the letter identification performance of each one of these methods on the artificially generated pseudo-real dataset. The pool of 70 P300 ERP waveforms, either obtained from the same subject in the passive-modality or from each subject in the active-modality are used to compose the artificial P300 wave in the pseudo-real dataset. Templates are randomly selected. 
- Experiment 2-Latency Noise: Instead of superimposing the P300 ERPs over the EEG trace at the exact locations where stimulus onsets are situated, an artificial latency lag is added. The lagging value is picked from a uniform distribution $U(0,0.4)$ [s] ranging from 0 to 0.4 of the $1 \mathrm{~s}$ segment size [74].

- Experiment 3-Component Amplitude Noise: the amplitude of the main P3b component of the ERP template is randomly altered. This component is defined to be located from the stimulus onset between $148 \mathrm{~ms}$ up to $996 \mathrm{~ms}$ which is around $840 \mathrm{~ms}$ long. This waveform element, multiplied by a gain factor, is subtracted from the original template. This gain factor between 0 and 1 is drawn from a uniform distribution $U(0,1)$. Additionally this subtracted waveform is multiplied by a Gaussian window with a support of the same length [75]. This avoids adding any discontinuity into the artificial generated signal.

All these experiments are executed using cross validation procedure dividing the letter to spell in two sets, preserving the structure of the letter identification trials. Spelling letters are scrambled while the order and group of each intensification sequence is preserved.

Finally the performance at letter identifications for these same methods is evaluated by running an offline BCI Simulation on the Dataset IIb of the BCI Competition II (2003) [76]. The protocol of this dataset is very similar to what was used to obtain the pseudo-real dataset. The sampling frequency of this dataset is 240 , the number of letters are 73 where the first 42 are used to create the template dictionary for all the methods and the remaining 31 are used to test the character recognition rate performance. Additionally, in this dataset the number of available intensification number sequences is 15. The classification method Support Vector Machine SVM with a linear kernel, is added for comparison as control using a feature $f$ constructed by normalizing the signal on each channel [77]. This method has been proved efficient in decoding P300 in several BCI Competitions [78].

\subsubsection{Classification}

The same classification algorithm based on k-nearest neighbors is used for all the methods [79]. The experimental protocol used to generated the pseudo-real dataset used in the experiments 1 to 3 is composed of 35 trials to spell 7 words of 5 letters each. Each trial is composed of 10 intensification sequences of the 6 columns and 6 rows of the Speller Matrix. Fifteen trials are used to build the dictionary of templates, extracting the averaged EEG segments for the row and column that already contain the P300 ERP, hence shielding 30 different templates per channel. Figure 5 shows the set of templates while using the first 15 trials of the dataset.

Described algorithms produce a feature $f$ for each averaged EEG segment. The aim of the classification procedure is to identify for the remaining 20 trials which of the 6 features $f$ that were obtained for row intensification, labeled by $\{1, \ldots, 6\}$, and which of the 6 features for column intensification, named $\{7, \ldots, 12\}$ are the ones that elicited the P300 response on the averaged EEG segment. The row number of the matrix can be obtained by doing

$$
r \hat{o w}=\arg \min _{u \in\{1, \ldots, 6\}} \sum_{i=1}^{k}\left\|f_{u}-q_{i}\right\|^{2}
$$

with $q_{i}$ being the set of k-nearest neighbors of the feature $f_{u}$ with $u$ varying from 1 to 6 . The parameter $k$ represents the number of neighbors chosen from the dictionary of templates. The column can be obtained in the same way,

$$
\hat{c o l} l=\arg \min _{u \in\{7, \ldots, 12\}} \sum_{i=1}^{k}\left\|f_{u}-q_{i}\right\|^{2} .
$$

Thus, the letter identification performance can be obtained by measuring the accuracy channel-by-channel at identifying the correct letter on the matrix, coordinated by rôtw and $\hat{c o l}$. 

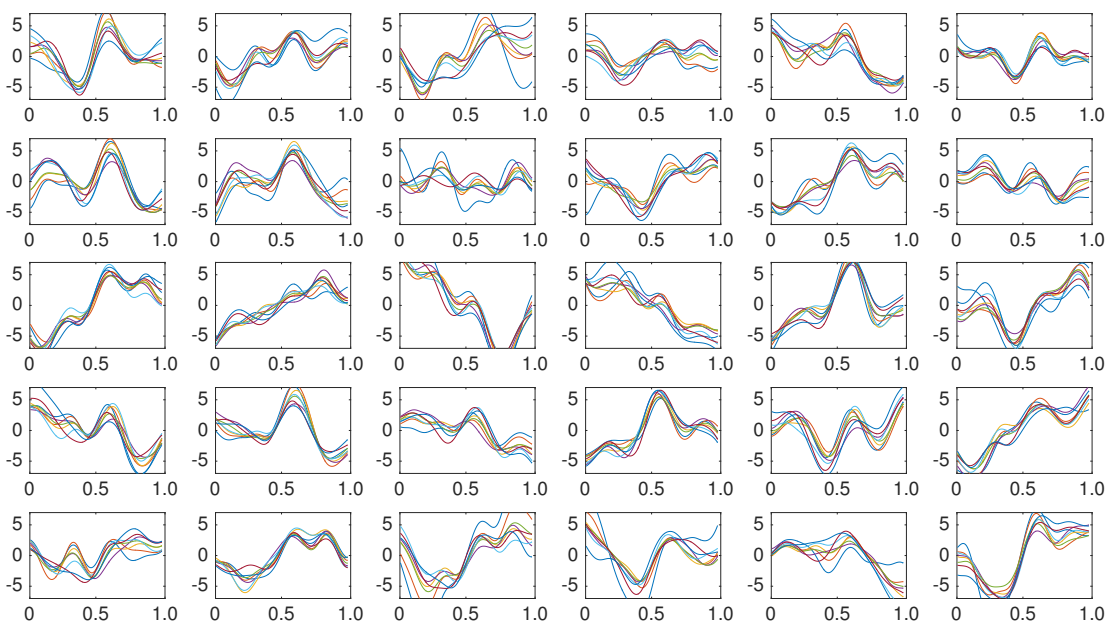

Figure 5. Coherently averaged signals segments of 1-second length containing the superimposed ERP. Vertical axis unit is $\mu \mathrm{V}$. Each one is extracted from the EEG signal of the Subject 1 of the pseudo-real dataset. These averaged signals correspond to the 15 first trials ( 2 averaged signals from each trial, one belonging to the column flashing and the other to row flashing). These are the templates used to build a dictionary per channel per subject and are used by the classification algorithm described in Section 3.6.5.

\section{Results}

Results for the first experiment are shown in Figures 6 and 7. The performance while identifying each letter of the standard P300 Speller Matrix, and the channels where the best and worst performance are attained, are shown. Each one represents the percentage of letters that is actually predicted by the algorithms using a cross-validation procedure. As previously described the data is continuously divided in two sets, where the first 15 letters are used to derive the dictionary of templates while the remaining 20 letters are used to measure the letter identification performance. This is repeated one hundred times, and performances averaged. Figure 6 shows the results for the passive modality while Figure 7 shows the results for the active modality.

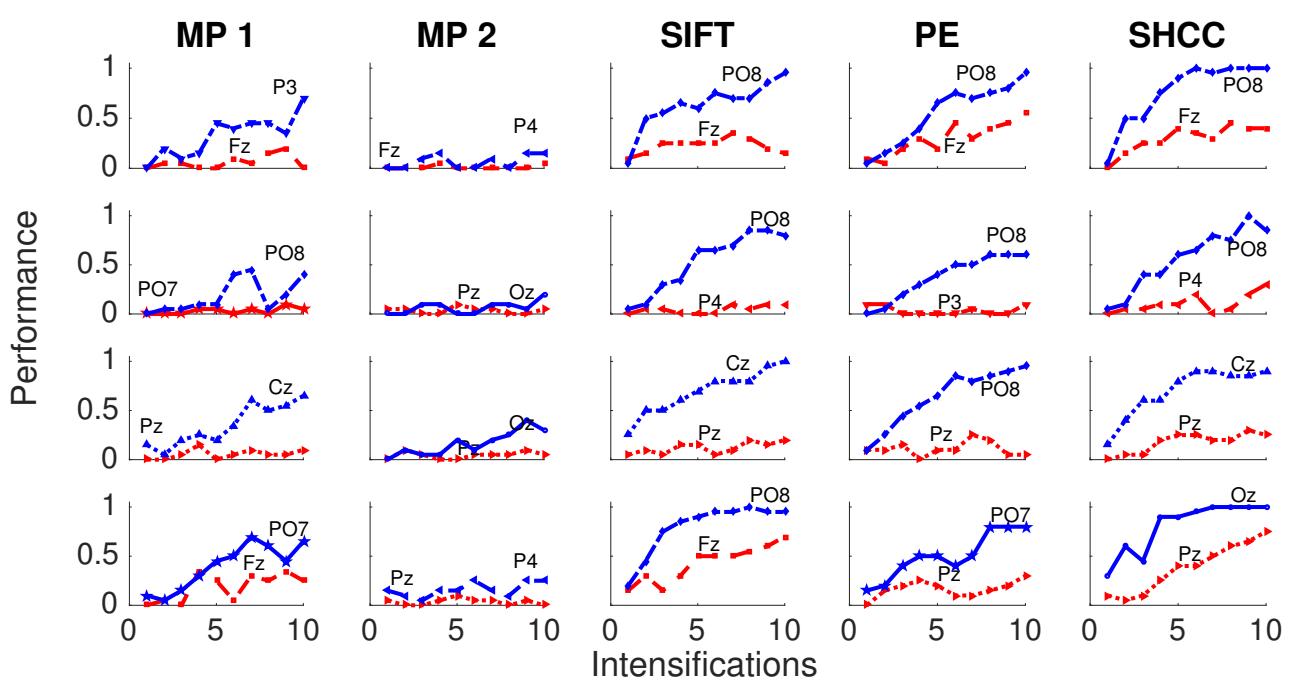

Figure 6. Passive Modality—Experiment 1: Speller performance curves obtained for each method for the four subjects that performed the passive modality protocol. Y-axis shows performance accuracy while $X$-axis shows the number of intensification sequences used to calculate the point-to-point signal average. The two curves show the performance for the best and worst performing channel. 


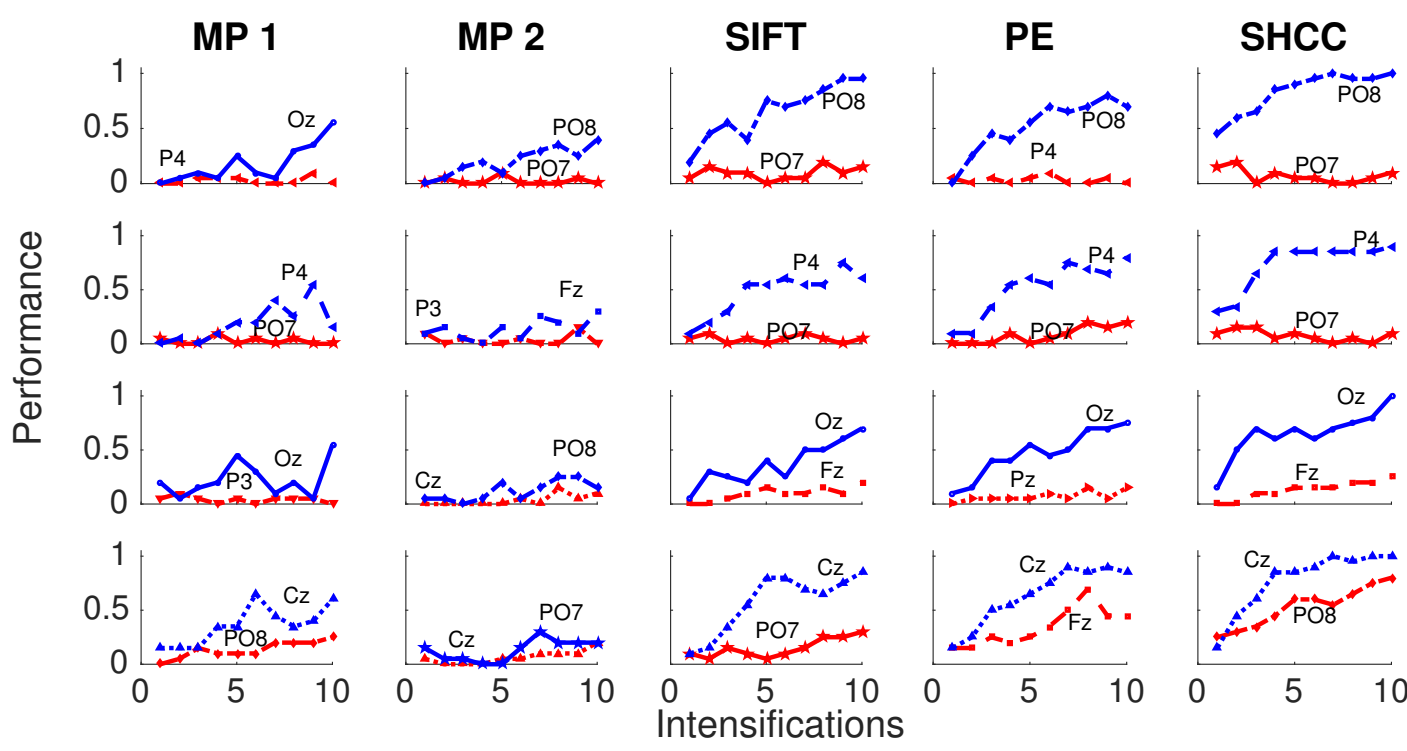

Figure 7. Active Modality-Experiment 1:Speller performance curves obtained for each method for the four subjects that performed the active modality protocol. Y-axis shows performance accuracy while $\mathrm{X}$-axis shows the number of intensification sequences used to calculate the point-to-point signal average. The two curves show the performance for the best and worst performing channel.

Figures 8 and 9 shows the performance curves for five algorithms for the second experiment, where a noisy latency lag is included. Best and worst channels are also shown.

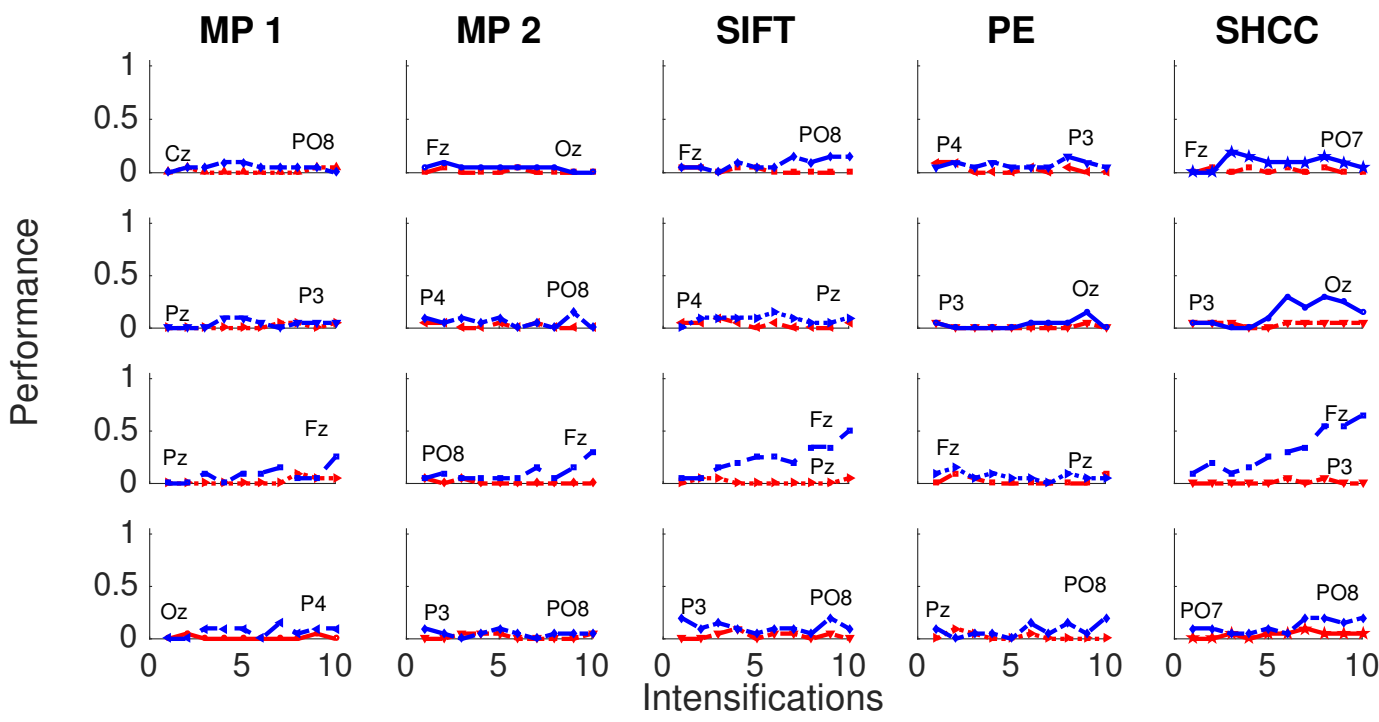

Figure 8. Passive Modality-Experiment 2: Performance curves for four subjects for the five algorithms when a random latency is included when superimposing the P300 signal template.

Finally, Figures 10 and 11 represents the performance values obtained for the Experiment 3, when the amplitude of the P3b component of the template is randomly attenuated.

Furthermore, results obtained for the dataset BCI Competition $2003 \mathrm{IIb}$ are shown in Figure 12 and in Table 2. For this experiment the number of available intensification sequences is 15. 
Table 2. Speller classification performance obtained for the dataset Ilb of the BCI Competition II (2003) for each one of the algorithms using 15 repetitions of intensification sequences. The first 42 trials are used for training to build the template dictionary and the remaining 31 for testing. The channel where the best performance is attained, is also shown.

\begin{tabular}{ccc}
\hline Method & Channel & Performance \\
\hline MP 1 & Cz & $50 \%$ \\
MP 2 & FC1 & $22 \%$ \\
SIFT & FC1 & $67 \%$ \\
PE & CP1 & $22 \%$ \\
SHCC & Cz & $61 \%$ \\
SVM & C1 & $32 \%$ \\
\hline
\end{tabular}

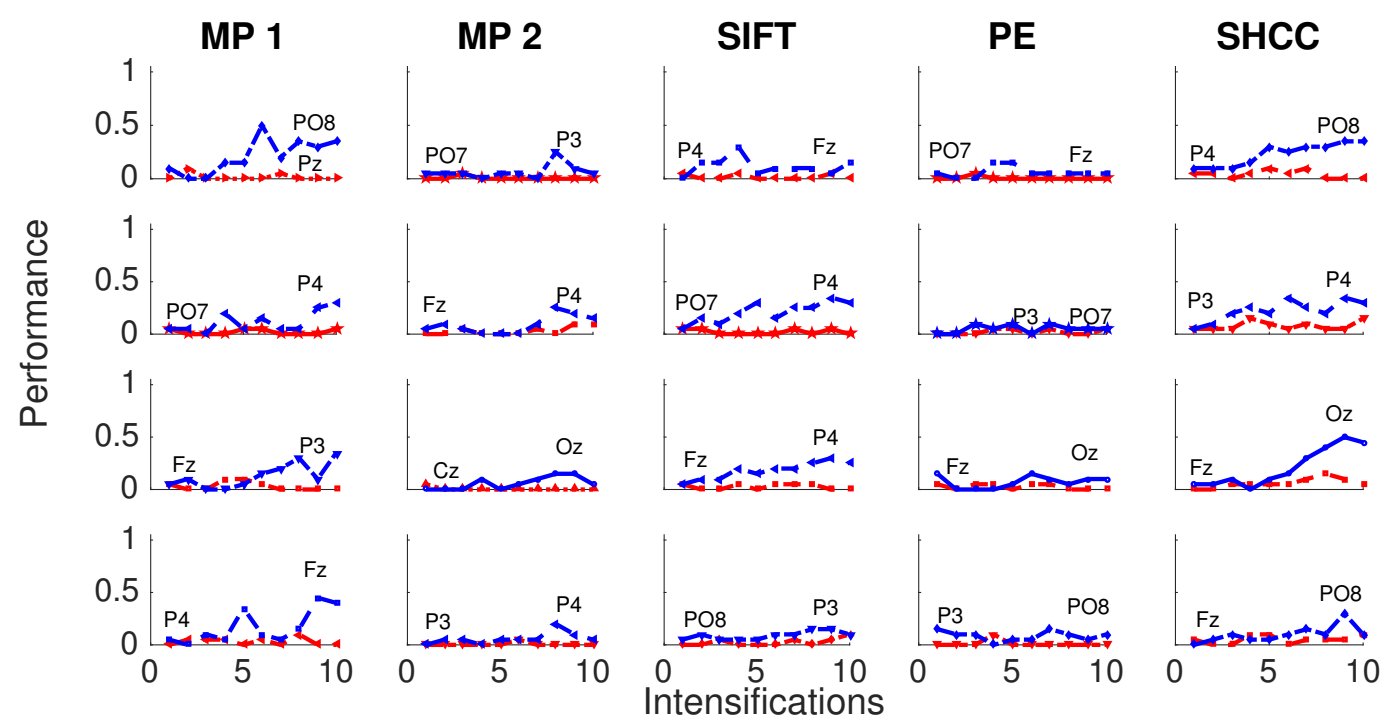

Figure 9. Active Modality-Experiment 2: Performance curves for the four subjects for the five algorithms. A random latency is included while superimposing the P300 signal template.

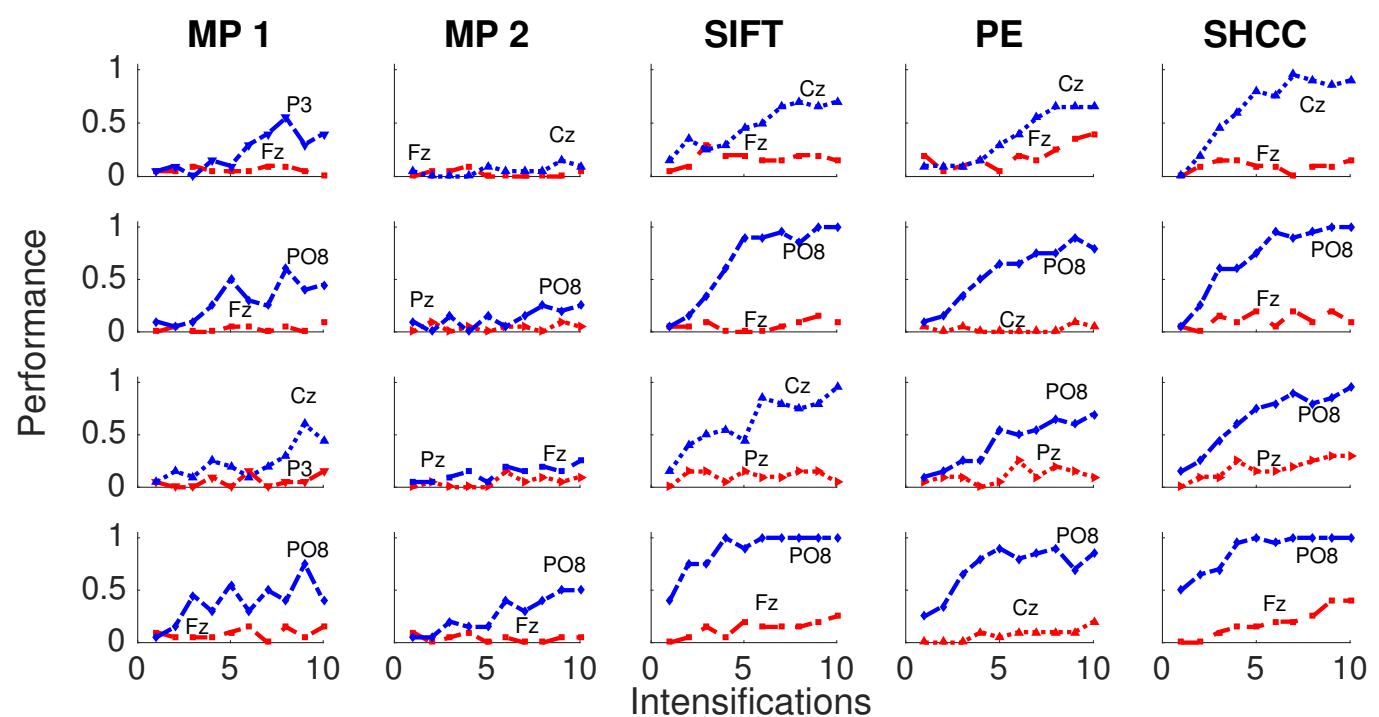

Figure 10. Passive Modality-Experiment 3:Performance curves for four subjects for the five algorithms when the amplitude of the P3b component of the template is randomly attenuated. 


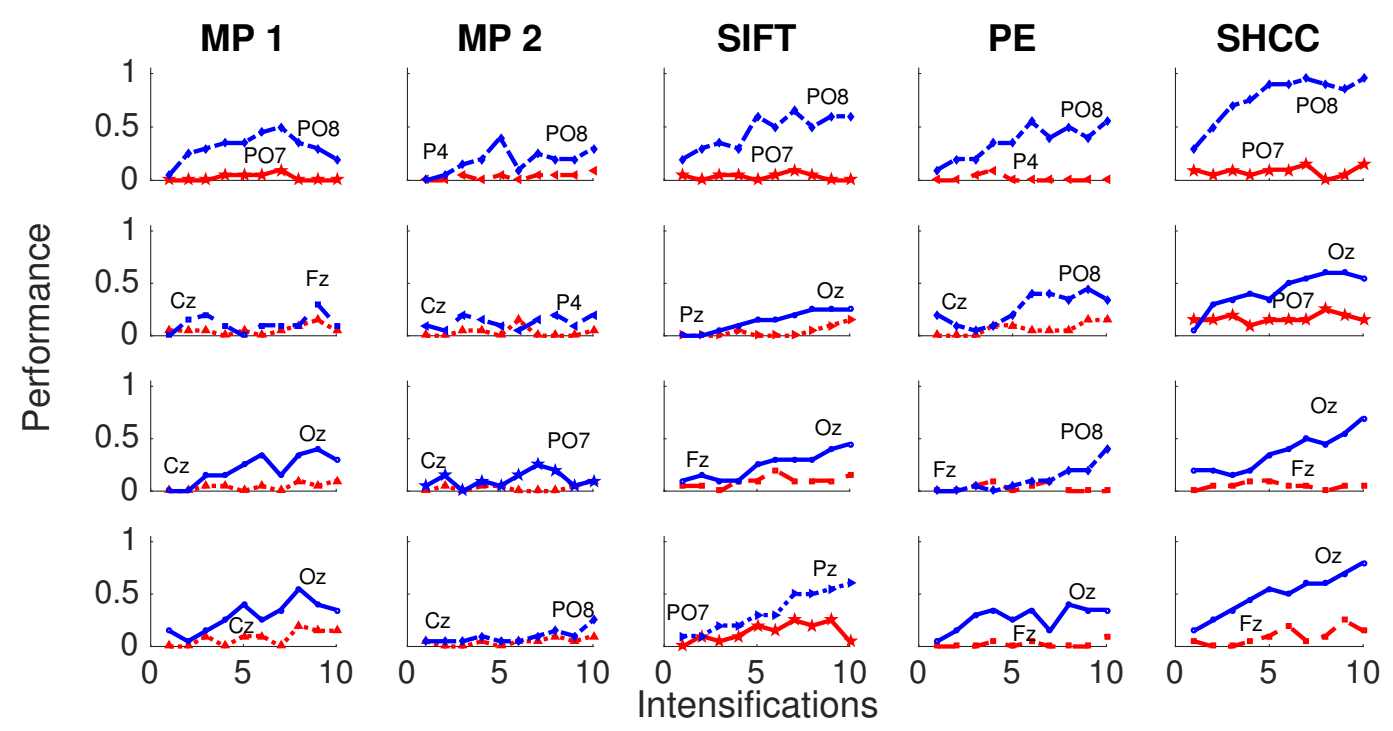

Figure 11. Active Modality-Experiment 3:Performance curves for four subjects for the five algorithms when the amplitude of the P3b component of the template is randomly attenuated.
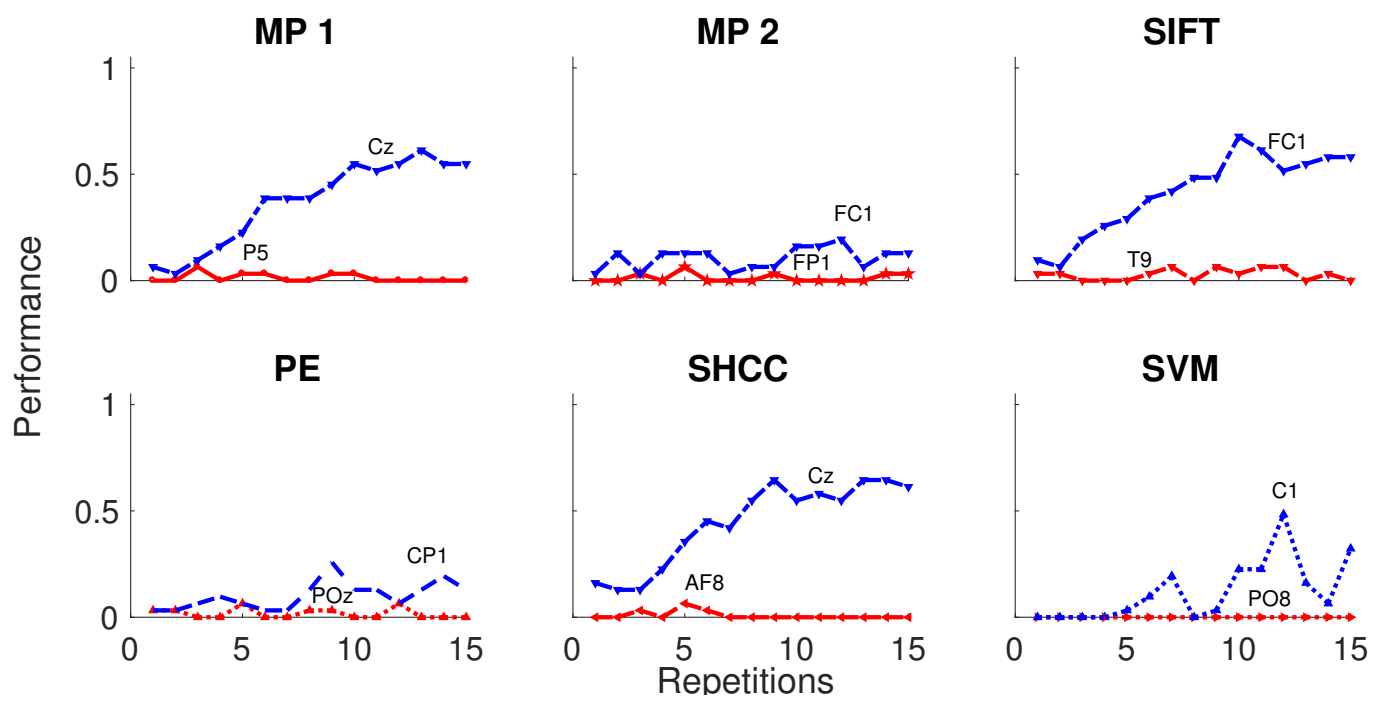

Figure 12. Speller performance obtained for the Dataset IIb of the BCI Competition II (2003) for each one of the algorithms. An offline BCI Simulation is performed using the first 42 trials as training and the remaining 31 as testing. The horizontal axis show the number of intensification sequences, from 0 to 15 for this dataset, while the vertical axis show the performance rate.

\section{Discussion}

A significant reduction of performance was found when latency noise is added. The latency noise reduces the information contained in the averaged signal, mainly due to the invalidation of the SNR enhancement performed by the signal averaging procedure. This reduction alters the obtained shape of the waveform of the ERP and impacts on the performances regardless of the method. On the other hand, all the algorithms show some resistance to noise in peak amplitudes of the main component. This is shown by the similarities of obtained results between the Experiment 1 and 3 .

Using a straightforward dictionary of templates for $M P-1$ proved more beneficial in terms of performance than the approach of using a Hilbert base of Wavelets atoms on MP-2. Either applying latency noise or amplitude noise, the method based on the signal's templates instead of using their coefficients achieve much better character identification rates. 
Regarding results produced for the public and real dataset IIb of P300 ERP from the Berlin BCI Competition II (2003), the obtained character identification rate is above theoretical chance level, and for some algorithms close to the usable threshold of $70 \%[80,81]$. When the character identification rate reaches this level of performance, the usage of word predicting algorithms allows to implement practical speller applications. Results for this competition have shown perfect classification with tailored algorithms [82]. This level is also similar to the performance obtained for the Experiment 3, which represents coincidentally the more realistic scenario for the pseudo-real dataset. It is important to remark that the algorithms presented here analyze the waveform structure of a single-channel signal $[65,83]$.

\section{Conclusions}

The purpose of this work is threefold, (1) raise awareness about the utility of using automatic waveform-based methods to study EEG signals, (2) to provide an overview of the state-of-the-art of those methods, and (3) to compare those methods and verify if it is possible to obtain acceptable classification performances based exclusively on the signal's waveform.

The higher performance results are obtained for the methods SHCC and SIFT either on the pseudo-real dataset and on the BCI Competition. We verified that it is possible to obtain discriminating information from the underlying signal based exclusively on an automated method of processing the waveforms. This brings the possibility to use these techniques to implement intelligible [84] automatic detection procedures, i.e., systems that are able to emphasize clearly and noticeable what are the factors that caused the system action, decision or classification. This is due to the fact that they are based on metrics which can be visually verified.

Further work should be conducted in terms of a multichannel meaningful extension of these waveform-based methods [83]. Moreover, the possibilities of finding overcomplete dictionaries for matching pursuit sparse representation based on obtained signal templates, could also be considered an area of future improvement.

We believe that the adoption of a hybrid methodology which can process the signal automatically, but at the same time, maintains an inherent intelligible property that can be mapped to existing procedures, and above all, can maintain the clinician trust on the system behavior is beneficial to Clinical Practice, Neuroscience and BCI research. Additionally, this may foster collaboration in a multidisciplinary environment and may ease the acceptance and translation of BCI technology [66]. The reason being, for caregivers and medical staff, particularly those with the expertise of the clinical EEG which is based on waveforms, they may feel a natural understanding of how the system is performing.

Another benefit of these methodologies is that they have a potential universal applicability. As they are only analyzing waveforms, they can be explored in other disciplines where the structure or shape of the waveform is of relevance. Analyzing signals by their waveforms is relative common in chemical analysis [85], seismic analysis in Geology [86], and quantitative financial analysis. Electrocardiogram EKG, on the other hand, has been extensively processed and studied analyzing the waveform structure [87].

Author Contributions: This projects is part of a R.R.'s PhD Thesis which is directed by J.M.S. and codirected by A.J.V.

Funding: This project was supported by the ITBACyT-15 funding program issued by ITBA University.

Acknowledgments: We would like to thank to Valentina Unakafova for providing the Permutation Entropy algorithm and to Montserrat-Alvarado González for providing the source code and a detailed description of the SHCC algorithm.

Conflicts of Interest: The authors declare no conflict of interest. 


\section{Abbreviations}

The following abbreviations are used in this manuscript:

$\begin{array}{ll}\text { EEG } & \text { electroencephalography } \\ \text { BCI } & \text { Brain Computer Interfaces } \\ \text { BMI } & \text { Brain Machine Interfaces } \\ \text { BNCI } & \text { Brain-Neural Computer Interfaces } \\ \text { SNR } & \text { Signal to Noise Ratio } \\ \text { CNS } & \text { Central Nervous System } \\ \text { AC } & \text { Alternating Current } \\ \text { DC } & \text { Direct Current } \\ \text { ERP } & \text { Event-Related Potential } \\ \text { P300 } & \text { Positive deflection at } 300 \text { ms } \\ \text { ITR } & \text { Information Transfer Rate } \\ \text { BTR } & \text { Bit Transfer Rate } \\ \text { SIFT } & \text { Scale Invariant Feature Transform } \\ \text { SHCC } & \text { Slope Horizontal Chain Code } \\ \text { PE } & \text { Permutation Entropy } \\ \text { MP } & \text { Matching Pursuit } \\ \text { ICU } & \text { Intensive Care Unit } \\ \text { EKG } & \text { Electrocardiogram } \\ \text { PAA } & \text { Period Amplitude Analysis } \\ \text { SVM } & \text { Support Vector Machine }\end{array}$

\section{References}

1. Wolpaw, J.R.; Birbaumer, N.; McFarland, D.J.; Pfurtscheller, G.; Vaughan, T.M. Brain-computer interfaces for communication and control. Clin. Neurophysiol. 2002, 113, 767-791. [CrossRef]

2. Lutz, W.; Sanderson, W.; Scherbov, S. The coming acceleration of global population ageing. Nature 2008, 451, 716-719. [CrossRef] [PubMed]

3. Domingo, M.C. An overview of the Internet of Things for people with disabilities. J. Netw. Comput. Appl. 2012, 35, 584-596. [CrossRef]

4. Guger, C.; Allison, B.Z.; Lebedev, M.A. Introduction. In Brain Computer Interface Research: A State of the Art Summary 6; Springer: Cham, Switzerland, 2017; pp. 1-8.

5. Schomer, D.L.; Silva, F.L.D. Niedermeyer's Electroencephalography: Basic Principles, Clinical Applications, and Related Fields; Oxford University Press: Oxford, UK, 2017.

6. Puce, A.; Hämäläinen, M.S. A review of issues related to data acquisition and analysis in EEG/MEG studies. Brain Sci. 2017, 7, 58. [CrossRef] [PubMed]

7. De Vos, M.; Debener, S. Mobile eeg: Towards brain activity monitoring during natural action and cognition. Int. J. Psychophysiol. 2014, 91, 1-2. [CrossRef] [PubMed]

8. Hartman, A.L. Atlas of EEG Patterns; Lippincott Williams \& Wilkins: Philadelphia, PA, USA, 2005.

9. Yuste, R.; Goering, S.; Agüeray Arcas, B.; Bi, G.; Carmena, J.M.; Carter, A.; Fins, J.J.; Friesen, P.; Gallant, J.; Huggins, J.E.; et al. Four ethical priorities for neurotechnologies and AI. Nature 2017, 551, 159-163. [CrossRef] [PubMed]

10. Tzimourta, K.D.; Tsoulos, I.; Bilero, T.; Tzallas, A.T.; Tsipouras, M.G.; Giannakeas, N. Direct Assessment of Alcohol Consumption in Mental State Using Brain Computer Interfaces and Grammatical Evolution. Inventions 2018, 3, 51. [CrossRef]

11. Kevric, J.; Subasi, A. The Impact of Mspca Signal De-Noising In Real-Time Wireless Brain Computer Interface System. Southeast Eur. J. Soft Comput. 2016, 4, 1-12. [CrossRef]

12. Cruz, A.; Pires, G.; Nunes, U.J. Double ErrP Detection for Automatic Error Correction in an ERP-Based BCI Speller. IEEE Trans. Neural Syst. Rehabil. Eng. 2018, 26, 26-36. [CrossRef] [PubMed]

13. Jansen, B.H. Quantitative analysis of electroencephalograms: Is there chaos in the future? Int. J. Bio-Med. Comput. 1991, 27, 95-123. [CrossRef] 
14. Thakor, N.V.; Tong, S. Advances in Quantitative Electroencephalogram Analysis Methods. Annu. Rev. Biomed. Eng. 2004, 6, 453-495. [CrossRef] [PubMed]

15. Jackson, A.F.; Bolger, D.J. The neurophysiological bases of EEG and EEG measurement: A review for the rest of us. Psychophysiology 2014, 51, 1061-1071. [CrossRef] [PubMed]

16. Haberman, M.A.; Spinelli, E.M. A multichannel EEG acquisition scheme based on single ended amplifiers and digital DRL. IEEE Trans. Biomed. Circuits Syst. 2012, 6, 614-618. [CrossRef] [PubMed]

17. Weeda, W.D.; Grasman, R.P.P.P.; Waldorp, L.J.; van de Laar, M.C.; van der Molen, M.W.; Huizenga, H.M. A fast and reliable method for simultaneous waveform, amplitude and latency estimation of single-trial EEG/MEG data. PLoS ONE 2012, 7, e38292. [CrossRef] [PubMed]

18. Farzan, F.; Atluri, S.; Frehlich, M.; Dhami, P.; Kleffner, K.; Price, R.; Lam, R.W.; Frey, B.N.; Milev, R.; Ravindran, A.; et al. Standardization of electroencephalography for multi-site, multi-platform and multi-investigator studies: Insights from the canadian biomarker integration network in depression. Sci. Rep. 2017, 7, 7473. [CrossRef] [PubMed]

19. Cole, S.R.; Voytek, B. Brain Oscillations and the Importance of Waveform Shape. Trends Cogn. Sci. 2017, 21,137-149. [CrossRef] [PubMed]

20. Buzsáki, G.; Anastassiou, C.A.; Koch, C. The origin of extracellular fields and currents-EEG, ECoG, LFP and spikes. Nat. Rev. Neurosci. 2012, 13, 407-420. [CrossRef] [PubMed]

21. Giagante, B.; Oddo, S.; Silva, W.; Consalvo, D.; Centurion, E.; D’Alessio, L.; Solis, P.; Salgado, P.; Seoane, E.; Saidon, P.; et al. Clinical-electroencephalogram patterns at seizure onset in patients with hippocampal sclerosis. Clin. Neurophysiol. 2003, 114, 2286-2293. [CrossRef]

22. Rodenbeck, A.; Binder, R.; Geisler, P.; Danker-Hopfe, H.; Lund, R.; Raschke, F.; Weeß, H.G.; Schulz, H. A review of sleep EEG patterns. Part I: A compilation of amended rules for their visual recognition according to Rechtschaffen and Kales. Somnologie 2006, 10, 159-175. [CrossRef]

23. Boostani, R.; Karimzadeh, F.; Nami, M. A comparative review on sleep stage classification methods in patients and healthy individuals. Comput. Methods Programs Biomed. 2017, 140, 77-91. [CrossRef] [PubMed]

24. Dimitriadis, S.I.; Salis, C.; Linden, D. A novel, fast and efficient single-sensor automatic sleep-stage classification based on complementary cross-frequency coupling estimates. Clin. Neurophysiol. 2018, 129, 815-828. [CrossRef] [PubMed]

25. Uchida, S.; Feinberg, I.; March, J.D.; Atsumi, Y.; Maloney, T. A comparison of period amplitude analysis and FFT power spectral analysis of all-night human sleep EEG. Physiol. Behav. 1999, 67, 121-131. [CrossRef]

26. Britton, J.W.; Frey, L.C.; Hopp, J.L.; Korb, P.; Koubeissi, M.Z.; Lievens, W.E.; Pestana-Knight, E.M.; St. Louis, E.K. Electroencephalography (EEG): An Introductory Text and Atlas of Normal and Abnormal Findings in Adults, Children, and Infants; American Epilepsy Society: Chicago, IL, USA, 2016.

27. Luck, S.J. An Introduction to the Event-Related Potential Technique; USA; MIT Press: Cambridge, MA, USA, 2005; Volume 78, p. 388.

28. Tatum, W.; Husain, A.; Benbadis, S.; Kaplan, P. Handbook of EEG Interpretation; Demos Medical Publishing: New York, NY, USA, 2008.

29. Cacioppo, J.; Tassinary, L.G.; Berntson, G.G. The Handbook of Psychophysiology; Cambridge University Press: Cambridge, UK, 2007.

30. Kappenman, E.S.; Luck, S.J. The Oxford Handbook of Event-Related Potential Components; Oxford University Press: Oxford, UK, 2012.

31. Ouyang, G.; Hildebrandt, A.; Sommer, W.; Zhou, C. Exploiting the intra-subject latency variability from single-trial event-related potentials in the $\mathrm{P} 3$ time range: A review and comparative evaluation of methods. Neurosci. Biobehav. Rev. 2017, 75, 1-21. [CrossRef] [PubMed]

32. Sanei, S.; Chambers, J. EEG Signal Processing; Wiley: Chichester, UK, 2007.

33. Wulsin, D.F.; Gupta, J.R.; Mani, R.; Blanco, J.A.; Litt, B. Modeling electroencephalography waveforms with semi-supervised deep belief nets: Fast classification and anomaly measurement. J. Neural Eng. 2011, 8, 036015, [CrossRef] [PubMed]

34. Hirsch, L.J.; Richard, B.P. Atlas of EEG in Critical Care; Wiley-Blackwell: Hoboken, NJ, USA, 2010; p. 348.

35. Subha, D.P.; Joseph, P.K.; Acharya U, R.; Lim, C.M. EEG signal analysis: A survey. J. Med. Syst. 2010, 34, 195-212. [CrossRef] [PubMed] 
36. Mak, J.N.; McFarland, D.J.; Vaughan, T.M.; McCane, L.M.; Tsui, P.Z.; Zeitlin, D.J.; Sellers, E.W.; Wolpaw, J.R. EEG correlates of P300-based brain-computer interface (BCI) performance in people with amyotrophic lateral sclerosis. J. Neural Eng. 2012, 9, 026014. [CrossRef] [PubMed]

37. Müller-Putz, G.R.; Riedl, R.; Wriessnegger, S.C. Electroencephalography (EEG) as a research tool in the information systems discipline: Foundations, measurement, and applications. Commun. Assoc. Inf. Syst. 2015, 37, 911-948. [CrossRef]

38. Shah, N.A.; Wusthoff, C.J. How to use: Amplitude-integrated EEG (aEEG). Arch. Dis. Child. Educ. Pract. Ed. 2015, 100, 75-81. [CrossRef] [PubMed]

39. Moher, D.; Liberati, A.; Tetzlaff, J.; Altman, D.G.; Altman, D.; Antes, G.; Atkins, D.; Barbour, V.; Barrowman, N.; Berlin, J.A.; et al. Preferred reporting items for systematic reviews and meta-analyses: The PRISMA statement. PLoS Med. 2009, 6, e1000097. [CrossRef] [PubMed]

40. Allen, R.L.; Mills, D. Signal Analysis: Time, Frequency, Scale, and Structure; John Wiley \& Sons: Hoboken, NJ, USA, 2004.

41. Wolpaw, J.; Wolpaw, E.W. Brain-Computer Interfaces: Principles and Practice; Oxford University Press: Oxford, UK, 2012; p. 400.

42. Thakor, N. Quantitative EEG Analysis Methods and Clinical Applications; Artech House Series Engineering in Medicine and Biology: Norwood, MA, USA, 2009; p. 440.

43. Jaškowski, P.; Verleger, R. An evaluation of methods for single-trial estimation of P3 latency. Psychophysiology 2000, 37, 153-162. [CrossRef] [PubMed]

44. Zhang, D.; Luo, Y. The P1 latency of single-trial ERPs estimated by two peak-picking strategies. In Proceedings of the 2011 4th International Conference on Biomedical Engineering and Informatics (BMEI), Shanghai, China, 15-17 October 2011; Volume 2, pp. 882-886.

45. Alvarado-González, M.; Garduño, E.; Bribiesca, E.; Yáñez-Suárez, O.; Medina-Bañuelos, V. P300 Detection Based on EEG Shape Features. Comput. Math. Methods Med. 2016, 2016, 1-14. [CrossRef] [PubMed]

46. Farwell, L.A.; Donchin, E. Talking off the top of your head: Toward a mental prosthesis utilizing event-related brain potentials. Electroencephalogr. Clin. Neurophysiol. 1988, 70, 510-523. [CrossRef]

47. Klein, F.F. A waveform analyzer applied to the human EEG. IEEE Trans. Biomed. Eng. 1976, 23, $246-252$. [CrossRef] [PubMed]

48. Yamaguchi, T.; Fujio, M.; Inoue, K.; Pfurtscheller, G. Design Method of Morphological Structural Function for Pattern Recognition of EEG Signals During Motor Imagery and Cognition. In Proceedings of the 2009 Fourth International Conference on Innovative Computing, Information and Control (ICICIC), Kaohsiung, Taiwan, 7-9 December 2009; pp. 1558-1561.

49. Uchida, S.; Matsuura, M.; Ogata, S.; Yamamoto, T.; Aikawa, N. Computerization of Fujimori's method of waveform recognition a review and methodological considerations for its application to all-night sleep EEG. J. Neurosci. Methods 1996, 64, 1-12. [CrossRef]

50. Vincent, E.; Deville, Y. Handbook of Blind Source Separation-Independent Component Analysis and Applications; Elsevier: Amsterdam, The Netherlands, 2010; p. 831.

51. Mallat, S.G.; Zhang, Z. Matching Pursuits With Time-Frequency Dictionaries. IEEE Trans. Signal Process. 1993, 41, 3397-3415. [CrossRef]

52. KS, S.C.; Mishra, A.; Shirhatti, V.; Ray, S. Comparison of Matching Pursuit Algorithm with Other Signal Processing Techniques for Computation of the Time-Frequency Power Spectrum of Brain Signals. J. Neurosci. 2016, 36, 3399-3408.

53. Cohen, M.X. Analyzing Neural Time Series Data: Theory and Practice; MIT Press: Cambridge MA, USA, 2014.

54. Vařeka, L. Matching pursuit for p300-based brain-computer interfaces. In Proceedings of the 2012 35th International Conference on Telecommunications and Signal Processing, Prague, Czech Republic, 3-4 July 2012; pp. 513-516.

55. Bandt, C.; Pompe, B. Permutation Entropy: A Natural Complexity Measure for Time Series. Phys. Rev. Lett. 2002, 88, 174102. [CrossRef] [PubMed]

56. Nicolaou, N.; Georgiou, J. Permutation entropy: A new feature for brain-computer interfaces. In Proceedings of the 2010 IEEE Biomedical Circuits and Systems Conference, BioCAS 2010, Paphos, Cyprus, 3-5 November 2010; pp. 49-52.

57. Keller, K.; Mangold, T.; Stolz, I.; Werner, J. Permutation Entropy: New Ideas and Challenges. Entropy 2017, 19, 134. [CrossRef] 
58. Unakafova, V.; Keller, K. Efficiently Measuring Complexity on the Basis of Real-World Data. Entropy 2013, 15, 4392-4415. [CrossRef]

59. Berger, S.; Schneider, G.; Kochs, E.F.; Jordan, D. Permutation entropy: Too complex a measure for EEG time series? Entropy 2017, 19, 692. [CrossRef]

60. Lowe, G. SIFT-The Scale Invariant Feature Transform. Int. J. Comput. Vis. 2004, 2, 91-110. [CrossRef]

61. Edelman, S.; Intrator, N.; Poggio, T. Complex cells and object recognition. 1997, unpublished manuscript.

62. Ramele, R.; Villar, A.J.; Santos, J.M. BCI classification based on signal plots and SIFT descriptors. In Proceedings of the 4th International Winter Conference on Brain-Computer Interface (BCI), Yongpyong, South, 22-24 February 2016; pp. 1-4.

63. Zhang, R.; Xu, P.; Guo, L.; Zhang, Y.; Li, P.; Yao, D. Z-Score Linear Discriminant Analysis for EEG Based Brain-Computer Interfaces. PLoS ONE 2013, 8, e74433. [CrossRef] [PubMed]

64. Vedaldi, A.; Fulkerson, B. VLFeat-An open and portable library of computer vision algorithms. Design 2010, 3, 1-4.

65. Rakotomamonjy, A.; Guigue, V. BCI Competition III: Dataset II-Ensemble of SVMs for BCI P300 Speller. IEEE Trans. Biomed. Eng. 2008, 55, 1147-1154. [CrossRef] [PubMed]

66. Chavarriaga, R.; Fried-Oken, M.; Kleih, S.; Lotte, F.; Scherer, R. Heading for new shores! Overcoming pitfalls in BCI design. Brain-Comput. Interfaces 2017, 4, 60-73. [CrossRef] [PubMed]

67. Quiroga, R.Q.; Garcia, H. Single-trial event-related potentials with wavelet denoising. Clin. Neurophysiol. 2003, 114, 376-390. [CrossRef]

68. Renard, Y.; Lotte, F.; Gibert, G.; Congedo, M.; Maby, E.; Delannoy, V.; Bertrand, O.; Lécuyer, A. OpenViBE: An Open-Source Software Platform to Design, Test, and Use Brain Computer Interfaces in Real and Virtual Environments. Presence Teleoper. Virtual Environ. 2010, 19, 35-53. [CrossRef]

69. Riccio, A.; Simione, L.; Schettini, F.; Pizzimenti, A.; Inghilleri, M.; Belardinelli, M.O.; Mattia, D.; Cincotti, F. Attention and P300-based BCI performance in people with amyotrophic lateral sclerosis. Front. Hum. Neurosci. 2013, 7, 732. [CrossRef] [PubMed]

70. Brunner, C.; Blankertz, B.; Cincotti, F.; Kübler, A.; Mattia, D.; Miralles, F.; Nijholt, A.; Otal, B. BNCI Horizon 2020-Towards a Roadmap for Brain/Neural Computer Interaction. Lect. Notes Comput. Sci. 2014, $8513,475-486$.

71. Rao, R.P.N. Brain-Computer Interfacing: An Introduction; Cambridge University Press: New York, NY, USA, 2013; p. 319.

72. Clerc, M.; Bougrain, L.; Lotte, F. Brain-Computer Interfaces, Technology and Applications 2 (Cognitive Science); ISTE Ltd. and Wiley: London, UK, 2016; p. 324.

73. Gramfort, A.; Luessi, M.; Larson, E.; Engemann, D.A.; Strohmeier, D.; Brodbeck, C.; Goj, R.; Jas, M.; Brooks, T.; Parkkonen, L.; et al. MEG and EEG data analysis with MNE-Python. Front. Neurosci. 2013, 7, 267. [CrossRef] [PubMed]

74. Da Pelo, P.; De Tommaso, M.; Monaco, A.; Stramaglia, S.; Bellotti, R.; Tangaro, S. Trial latencies estimation of event-related potentials in EEG by means of genetic algorithms. J. Neural Eng. 2018, 15, 026016. [CrossRef] [PubMed]

75. Harris, F.J. On the Use of Windows for Harmonic Analysis with the Discrete Fourier Transform. Proc. IEEE 1978, 66, 51-83. [CrossRef]

76. Blankertz, B. Documentation Second Wadsworth BCI Dataset (P300 Evoked Potentials) Data Acquired Using BCI2000 P300 Speller Paradigm. BCI Classification Contest November. Available online: http: / /www.bbci.de/competition/ii/albany_desc/albany_desc_ii.pdf (accessed on 14 November 2018).

77. Krusienski, D.J.; Sellers, E.W.; Cabestaing, F.; Bayoudh, S.; McFarland, D.J.; Vaughan, T.M.; Wolpaw, J.R. A comparison of classification techniques for the P300 Speller. J. Neural Eng. 2006, 3, 299-305. [CrossRef] [PubMed]

78. Kaper, M.; Meinicke, P.; Grossekathoefer, U.; Lingner, T.; Ritter, H. BCI competition 2003-Data set IIb: Support vector machines for the P300 speller paradigm. IEEE Trans. Biomed. Eng. 2004, 51, 1073-1076. [CrossRef] [PubMed]

79. Boiman, O.; Shechtman, E.; Irani, M. In defense of nearest-neighbor based image classification. In Proceedings of the 26th IEEE Conference on Computer Vision and Pattern Recognition (CVPR), Anchorage, AK, USA, 23-28 June 2008. 
80. Käthner, I.; Halder, S.; Hintermüller, C.; Espinosa, A.; Guger, C.; Miralles, F.; Vargiu, E.; Dauwalder, S.; Rafael-Palou, X.; Solà, M.; et al. A multifunctional brain-computer interface intended for home use: An evaluation with healthy participants and potential end users with dry and gel-based electrodes. Front. Neurosci. 2017, 11, 286. [CrossRef] [PubMed]

81. Neuper, C.; Müller, G.; Kübler, A.; Birbaumer, N.; Pfurtscheller, G. Clinical application of an EEG-based brain-computer interface: A case study in a patient with severe motor impairment. Clin. Neurophysiol. 2003, 114, 399-409. [CrossRef]

82. Kundu, S.; Ari, S. P300 Detection with Brain-Computer Interface Application Using PCA and Ensemble of Weighted SVMs. IETE J. Res. 2018, 64, 406-414. [CrossRef]

83. Gribonval, R.; Rauhut, H.; Schnass, K.; Vandergheynst, P. Atoms of all channels, unite! Average case analysis of multi-channel sparse recovery using greedy algorithms. J. Fourier Anal. Appl. 2008, 14, 655-687. [CrossRef]

84. Bragg, M.J. The Challenge of Crafting Intelligible Intelligence. In Proceedings of the ACM Symposium on User Interface Software and Technology UIST 2018, Berlin, Germany, 14-17 October 2018.

85. Skoog, D.A.; West, D.M.; Holler, F.J.; Crouch, S.R. Analytical Chemistry: An Introduction; Saunders College Publishing: Philadelphia, PA, USA, 2000.

86. Owens, T.J.; Zandt, G.; Taylor, S.R. Seismic evidence for an ancient rift beneath the Cumberland Plateau, Tennessee: A detailed analysis of broadband teleseismic P waveforms. J. Geophys. Res. Solid Earth 1984, 89, 7783-7795. [CrossRef]

87. Stockman, G.; Kanal, L.; Kyle, M. Structural pattern recognition of carotid pulse waves using a general waveform parsing system. Commun. ACM 1976, 19, 688-695. [CrossRef]

(C) 2018 by the authors. Licensee MDPI, Basel, Switzerland. This article is an open access article distributed under the terms and conditions of the Creative Commons Attribution (CC BY) license (http:/ / creativecommons.org/licenses/by/4.0/). 